\title{
COMPUTING THE SINGULARITIES OF RATIONAL SURFACES
}

\author{
S. PÉREZ-DÍAZ, J. R. SENDRA, AND C. VILLARINO
}

\begin{abstract}
Given a rational projective parametrization $\mathcal{P}(\mathfrak{s}, \mathfrak{t}, \mathfrak{v})$ of a rational projective surface $\mathcal{S}$ we present an algorithm such that, with the exception of a finite set (maybe empty) $\mathfrak{B}$ of projective base points of $\mathcal{P}$, decomposes the projective parameter plane as $\mathbb{P}^{2}(\mathbb{K}) \backslash \mathfrak{B}=\bigcup_{k=1}^{\ell} \mathfrak{S}_{k}$ such that, if $\left(\mathfrak{s}_{0}: \mathfrak{t}_{0}:\right.$ $\left.\mathfrak{v}_{0}\right) \in \mathfrak{S}_{k}$, then $\mathcal{P}\left(\mathfrak{s}_{0}, \mathfrak{t}_{0}, \mathfrak{v}_{0}\right)$ is a point of $\mathcal{S}$ of multiplicity $k$.
\end{abstract}

\section{INTRODUCTION}

The study, analysis and computation of the singular locus of algebraic varieties is an old but still very active research topic. The interest in the study of singularities is motivated for several reasons, one being their applicability; for instance, in geometric modeling, when determining the shape and the topology of curves (either planar or spatial) and of surfaces, when computing surface integrals, etc. In this paper, we focus on the problem of computing the singularities, as well as their multiplicities, of rational surfaces given parametrically.

When the algebraic variety is given as a zero set of finitely many polynomials, the singularities and their multiplicities can be directly computed by applying elimination theory techniques as Gröbner bases, characteristic sets, etc. However, when the algebraic variety is unirational and it is given by means of a rational parametrization, besides the question of computing the singular locus and its multiplicity structure, one has the additional problem of determining the parameter values that generate the singular points with their corresponding multiplicities. This, for instance, can be useful when using a parametrization for plotting a curve or a surface or when utilizing a parametrization for analyzing the intersection variety of two varieties, one of them being parametrically given. Of course, one can always apply elimination techniques to first provide the defining implicit polynomials of the variety, second to determine the singularities from these polynomials, third to decompose the singular locus w.r.t. the multiplicities, and finally to compute the fibre (w.r.t. the parametrization) of the elements in the singular locus. Nevertheless, this can be inefficient because of the computational complexity.

So the challenge, in the unirational case, is to derive the singularities and their multiplicity directly from a parametric representation avoiding the computation of the ideal of the variety (for the notion of singularity and multiplicity see e.g. [2], Lectures 14 and 20). The case of rational curves (both planar and spatial) has been

Received by the editor December 25, 2011 and, in revised form, January 23, 2013, June 5, 2013, and October 27, 2013.

2010 Mathematics Subject Classification. Primary 14Q10; Secondary 14J17, 68W30.

This work was partially supported by the Spanish Ministerio de Ciencia e Innovación under the project MTM2008-04699-C03-01 and by the Ministerio de Economía y Competitividad under the project MTM2011-25816-C02-01; the authors are members of the Research Group ASYNACS (Ref. CCEE2011/R34). 
addressed by several authors (see [1, 4, [5], 10]). However, the case of rational surfaces has not been so extensively studied. We refer the reader to [3] where the case of rational ruled surfaces is analyzed.

In this paper, we present an algorithm for computing the singularities of a rational projective surface from an input rational projective parametrization not necessarily proper (i.e., birational). More precisely, the problem we deal with is stated as:

\section{Problem statement.}

- Given a rational projective parametrization

$$
\mathcal{P}(\mathfrak{s}, \mathfrak{t}, \mathfrak{v})=\left(\mathfrak{p}_{1}(\mathfrak{s}, \mathfrak{t}, \mathfrak{v}): \cdots: \mathfrak{p}_{4}(\mathfrak{s}, \mathfrak{t}, \mathfrak{v})\right),
$$

of a rational projective surface $\mathcal{S} \subset \mathbb{P}^{3}(\mathbb{K})$, where $\mathbb{K}$ is an algebraically closed field of characteristic zero.

- Decompose $\mathbb{P}^{2}(\mathbb{K}) \backslash \mathfrak{B}$, where $\mathfrak{B}=\left\{\bar{\alpha} \in \mathbb{P}^{2}(\mathbb{K}) \mid \mathfrak{p}_{1}(\bar{\alpha})=\cdots=\mathfrak{p}_{4}(\bar{\alpha})=0\right\}$, as

$$
\mathbb{P}^{2}(\mathbb{K}) \backslash \mathfrak{B}=\bigcup_{k=1}^{\ell} \mathfrak{S}_{k}
$$

such that, if $\left(\mathfrak{s}_{0}: \mathfrak{t}_{0}: \mathfrak{v}_{0}\right) \in \mathfrak{S}_{k}$, then $\mathcal{P}\left(\mathfrak{s}_{0}, \mathfrak{t}_{0}, \mathfrak{v}_{0}\right)$ is a point of $\mathcal{S}$ of multiplicity $k$.

Therefore, the output of the algorithm solving this problem will be a finite collection of quasiprojective varieties in $\mathbb{P}^{2}(\mathbb{K})$ (in the sense of [13], p. 46), namely $\left\{\mathfrak{S}_{i}\right\}$, described by its equations, such that the parameter values in each set generate points on the surface with the same multiplicity.

Although abusing the terminology, we will use the following definition.

Definition 1.1. The elements in $\mathfrak{S}_{1}$ are called $\mathcal{P}$-simple points of $\mathcal{S}$, and the elements in $\mathfrak{S}_{k}$, with $k>1, \mathcal{P}$-singularities of $\mathcal{S}$ of multiplicity $k$. We refer to these points as affine (either $\mathcal{P}$-simple or $\mathcal{P}$-singular) points if $\mathfrak{v}_{0} \neq 0$ and points (either $\mathcal{P}$-simple or $\mathcal{P}$-singular) at infinity if $\mathfrak{v}_{0}=0$. Moreover, we represent the multiplicity of $\left(\mathfrak{s}_{0}: \mathfrak{t}_{0}: \mathfrak{v}_{0}\right)$ as mult $\left(\left(\mathfrak{s}_{0}: \mathfrak{t}_{0}: \mathfrak{v}_{0}\right)\right)$ meaning

$$
\left.\operatorname{mult}\left(\left(\mathfrak{s}_{0}: \mathfrak{t}_{0}: \mathfrak{v}_{0}\right)\right)=\operatorname{mult}\left(\mathcal{P}\left(\mathfrak{s}_{0}, \mathfrak{t}_{0}, \mathfrak{v}_{0}\right), \mathcal{S}\right)\right)
$$

where $\operatorname{mult}(A, \mathcal{S})$ denotes the multiplicity of $A \in \mathbb{P}^{3}(\mathbb{K})$ w.r.t. $\mathcal{S}$.

The polynomials $\mathfrak{p}_{1}, \ldots, \mathfrak{p}_{4}$ are assumed to be homogeneous of the same degree and coprime. Therefore the parametrization $\mathcal{P}(\mathfrak{s}, \mathfrak{t}, \mathfrak{v})$ induces the regular map

$$
\begin{array}{clc}
\mathcal{P}: \quad \mathbb{P}^{2}(\mathbb{K}) \backslash \mathfrak{B} & \rightarrow & \mathcal{S} \\
\bar{\alpha} & \mapsto & \mathcal{P}(\bar{\alpha})
\end{array}
$$

where $\mathfrak{B}$ is as above, in the problem description; we call the elements in $\mathfrak{B}$ the (projective) base points of the parametrization (see Section 2). We will be able to decompose, as above, $\mathbb{P}^{2}(\mathbb{K}) \backslash \mathfrak{B}$.

$\mathfrak{B}$ is either zero-dimensional or empty. So, we will be missing (at most) finitely many parameter values in $\mathbb{P}^{2}(\mathbb{K})$. On the other hand, if $\mathfrak{B}=\emptyset$, since $\mathcal{S}$ is irreducible and $\mathcal{P}$ regular, then $\mathcal{P}\left(\mathbb{P}^{2}(\mathbb{K})\right)=\mathcal{S}$ (see e.g. Theorem 2, page 57, in [13]). Therefore, if $\mathfrak{B}=\emptyset$, our method will determine all singularities of $\mathcal{S}$. However, if $\mathfrak{B} \neq \emptyset$ the method will generate all singularities in the dense set $\mathcal{P}\left(\mathbb{P}^{2}(\mathbb{K}) \backslash \mathfrak{B}\right) \subset \mathcal{S}$. For avoiding this deficiency one may consider reparametrizing normally the parametrization, 
however, this is not an easy task (see 9]). We do not deal with this issue in this paper.

Our method is based on the generalization of the ideas in [5] in combination with the results in [6] and [7] that perform the computations without implicitizing. Intuitively speaking, the method works as follows; see Section 2 for further details. First we state a general formula for computing the multiplicity of an affine point of $\mathbb{K}^{3}$ w.r.t. an affine surface (see Section 3). Then, we study how this formula behaves under specializations when applied to a parametric (rational) point (see Section 4) and we derive an algorithm. Next, we analyze, by means of the algorithm in Section 44 the multiplicity of the (affine) parameter values of the form $p_{a}:=\left(\mathfrak{s}_{0}: \mathfrak{t}_{0}: 1\right)$ to later study the parameter values (at infinity) of the form $p_{\infty}:=\left(\mathfrak{s}_{0}: \mathfrak{t}_{0}: 0\right)$. In order to compute $\operatorname{mult}\left(p_{a}\right)$ we consider the four affine rational parametrizations (we call them $\left.\mathcal{P}_{1}, \ldots, \mathcal{P}_{4}\right)$ generated by $\mathcal{P}(\mathfrak{s}, \mathfrak{t}, \mathfrak{v})$ by dehomogenizing w.r.t. the first, second, third and fourth component of the parametrization, respectively and taking $\mathfrak{v}=1$. Then, we apply the algorithm in Section 4 to $p_{a}$ via one of the affine parametrizations, say, $\mathcal{P}_{4}$. This first attempt will classify all affine parameter values with the exception of a proper closed set, and hence with the exception of finitely many components of dimension either 1 or 0 . By using consecutively the other three affine parametrizations we achieve the multiplicity of all affine parameter values not covered by $\mathcal{P}_{4}$ and not being base points (see Section 5 ). Finally we deal with $p_{\infty}$ with a similar strategy but dehomogenizing with either $\mathfrak{s}=1$ or $\mathfrak{t}=1$.

The structure of the paper is as follows. In Section 2 we introduce the notation and we briefly describe the general strategy as well as the underlying ideas. In Section 3 we state the multiplicity formula, we develop a method for computing a point not on the surface (this will be needed in the algorithm), starting from the parametric representation and without implicitizing. Moreover, we briefly recall some procedures from [6] and 7]. In Section [4, we analyze the execution of the multiplicity formula for the case of rational parametric points, and we derive an algorithm. In Section [5, we deal with the determination of the $\mathcal{P}$-singularities, and it ends with the final algorithm that summarizes all the ideas derived previously. In Section 6, we illustrate the algorithm by means of two examples. Computations in the examples were carried out with the mathematical software Maple.

\section{NotAtion AND STRATEGy DESCRIPTION}

In this section we introduce the notation that will be used throughout the paper, we describe the general strategy, and we give the intuitive ideas underlining our reasoning.

$\mathbb{K}$ is an algebraically closed field of characteristic zero, and $\mathbb{P}^{2}(\mathbb{K}), \mathbb{P}^{3}(\mathbb{K})$ are the projective plane and projective space over $\mathbb{K}$, respectively. Let $\left(x_{1}: x_{2}: x_{3}: x_{4}\right)$ be the projective coordinates in $\mathbb{P}^{3}(\mathbb{K})$. In addition, throughout the paper, we introduce the following notation:

- $\mathcal{S} \subset \mathbb{P}^{3}(\mathbb{K})$ is a rational projective surface. For simplicity in the explanation, we assume that $\mathcal{S}$ is not a plane. Note that this assumption is not a loss of generality, because one can easily deduce whether a parametrically given surface is a plane, and in this case all points are simple.

- $\mathcal{P}(\mathfrak{s}, \mathfrak{t}, \mathfrak{v})=\left(\mathfrak{p}_{1}(\mathfrak{s}, \mathfrak{t}, \mathfrak{v}): \mathfrak{p}_{2}(\mathfrak{s}, \mathfrak{t}, \mathfrak{v}): \mathfrak{p}_{3}(\mathfrak{s}, \mathfrak{t}, \mathfrak{v}): \mathfrak{p}_{4}(\mathfrak{s}, \mathfrak{t}, \mathfrak{v})\right)$ is a rational projective parametrization of $\mathcal{S}$, where $\operatorname{gcd}\left(\mathfrak{p}_{1}, \mathfrak{p}_{2}, \mathfrak{p}_{3}, \mathfrak{p}_{4}\right)=1$ and the four 
polynomials are homogeneous (note that none of them is zero) of the same degree.

- $\mathcal{S}_{i}$ is the affine surface obtained by dehomogenization of $\mathcal{S}$ with $x_{i}=1$; note that $\mathcal{S}$ is not the plane $x_{i}=0$,

- and $\mathcal{P}_{i}(s, t)$ is the affine parametrization of $\mathcal{S}_{i}$ obtained by taking $\mathfrak{v}=1$ and dehomogenizing $\mathcal{P}(\mathfrak{s}, \mathfrak{t}, \mathfrak{v})$ w.r.t. the $i$-component.

- We say that $\left(\mathfrak{s}_{0}: \mathfrak{t}_{0}: \mathfrak{v}_{0}\right) \in \mathbb{P}^{2}(\mathbb{K})$ is a (projective) base point of $\mathcal{P}(\mathfrak{s}, \mathfrak{t}, \mathfrak{v})$ if $\mathfrak{p}_{1}\left(\mathfrak{s}_{0}, \mathfrak{t}_{0}, \mathfrak{v}_{0}\right)=\mathfrak{p}_{2}\left(\mathfrak{s}_{0}, \mathfrak{t}_{0}, \mathfrak{v}_{0}\right)=\mathfrak{p}_{3}\left(\mathfrak{s}_{0}, \mathfrak{t}_{0}, \mathfrak{v}_{0}\right)=\mathfrak{p}_{4}\left(\mathfrak{s}_{0}, \mathfrak{t}_{0}, \mathfrak{v}_{0}\right)=0$. We denote by $\mathfrak{B}$ the set of (projective) base points of $\mathcal{P}(\mathfrak{s}, \mathfrak{t}, \mathfrak{v})$.

- Furthermore, we denote by $\mathfrak{B}_{a}$ the set $\mathfrak{B}_{a}=\left\{\left(s_{0}, t_{0}\right) \in \mathbb{K}^{2} \mid\left(s_{0}: t_{0}: 1\right) \in\right.$ $\mathfrak{B}\}$. We call the points in $\mathfrak{B}_{a}$ the (affine) base points of $\mathcal{P}(\mathfrak{s}, \mathfrak{t}, \mathfrak{v})$. Often, we will consider $\mathfrak{B}_{a}$ naturally embedded in $\mathfrak{B}$.

We start with two technical lemmas on base points. Since $\mathfrak{B}$ is the intersection of the projective curves defined by $\mathfrak{p}_{i}(\mathfrak{s}, \mathfrak{t}, \mathfrak{v})$, and since $\operatorname{gcd}\left(\mathfrak{p}_{1}, \mathfrak{p}_{2}, \mathfrak{p}_{3}, \mathfrak{p}_{4}\right)=1$, we get the following lemma.

Lemma 2.1. $\operatorname{Card}(\mathfrak{B})<\infty$.

$\mathfrak{B}_{a}$ can be expressed in terms of the zeros of the denominators of $\mathcal{P}_{i}(s, t)$; when we will speak about the numerator or the denominator of a rational function we will always assume that the rational function is given in reduced form.

Lemma 2.2. $\mathfrak{B}_{a}=\bigcap_{i=1}^{4} \Delta_{i}$ where $\Delta_{i}$ is the algebraic set defined, over $\mathbb{K}$, by the least common multiple of the denominators of $\mathcal{P}_{i}(s, t)$.

Proof. Let $\mathcal{P}_{i}(s, t)=\left(p_{i, 1} / q_{i, 1}, p_{i, 2} / q_{i, 2}, p_{i, 3} / q_{i, 3}\right)$, with $\operatorname{gcd}\left(p_{i, j}, q_{i, j}\right)=1$, and let $\delta_{i}:=\operatorname{lcm}\left(q_{i, 1}, q_{i, 2}, q_{i, 3}\right)$. Let $\mathcal{P}_{i}(s, t)$ be also expressed as $\mathcal{P}_{i}(s, t)=\left(a_{i} / d_{i}, b_{i} / d_{i}\right.$, $\left.c_{i} / d_{i}\right)$ with $\operatorname{gcd}\left(a_{i}, b_{i}, c_{i}, d_{i}\right)=1$. We prove that $\delta_{i}=d_{i}$, and from there the proof is trivial. Let us assume that $\delta_{1} \neq d_{1}$; similarly for the others. Clearly $\delta_{1}$ divides $d_{1}$. So, there exists a non-trivial factor $H_{1}$ such that $d_{1}=\delta_{1} H_{1}$. Therefore, by construction, $H_{1}$ divides $d_{k}$, with $k=2,3,4$. However, $\operatorname{since} \operatorname{gcd}\left(\mathfrak{p}_{1}, \mathfrak{p}_{2}, \mathfrak{p}_{3}, \mathfrak{p}_{4}\right)=1$, then $\operatorname{gcd}\left(d_{1}, \ldots, d_{4}\right)=1$.

Furthermore, if $\Theta: \mathbb{K}^{n} \rightarrow \mathbb{K}^{m}$ is a rational affine map, we denote by $\operatorname{deg}(\Theta)$ the degree of the map $\Theta$ (see e.g. [13] p. 143, or [2] p. 80). In particular, $\operatorname{deg}\left(\mathcal{P}_{i}\right)$ denotes the degree of the rational map $\mathcal{P}_{i}$ induced by the rational parametrization $\mathcal{P}_{i}(s, t)$; that is, of the rational map

$$
\begin{aligned}
\mathcal{P}_{i}: \quad \mathbb{K}^{2} \backslash \Delta_{i} & \rightarrow \mathcal{S}_{i} \subset \mathbb{K}^{3} \\
\left(s_{0}, t_{0}\right) & \mapsto \mathcal{P}_{i}\left(s_{0}, t_{0}\right)
\end{aligned}
$$

where $\Delta_{i}$ is as in Lemma 2.2 .

Also, for a rational function $\chi$ we denote by $\operatorname{Numer}(\chi)$ the numerator of $\chi$. By $\operatorname{PrimPart}_{\left\{v_{1}, \ldots, v_{n}\right\}}(f)$ and Content ${ }_{\left\{v_{1}, \ldots, v_{n}\right\}}(f)$, where $f \in \mathbb{K}\left[x_{1}, \ldots, x_{m}\right]\left[v_{1}, \ldots, v_{n}\right]$, we denote the primitive part and the content w.r.t. $\left\{v_{1}, \ldots, v_{n}\right\}$ of $f$, respectively. That is, Content ${ }_{\left\{v_{1}, \ldots, v_{n}\right\}}(f)$ is the gcd of all the coefficients of $f$ w.r.t. $\left\{v_{1}, \ldots, v_{n}\right\}$, that are multivariate polynomials in $\mathbb{K}\left[x_{1}, \ldots, x_{m}\right]$, and $\operatorname{PrimPart}_{\left\{v_{1}, \ldots, v_{n}\right\}}(f)$ is $f /$ Content $_{\left\{v_{1}, \ldots, v_{n}\right\}}(f)$. In addition, for $f, g$ polynomials depending on $s$ we denote $\operatorname{Res}_{s}(f, g)$ its resultant.

For $P=\left(a_{1}: a_{2}: a_{3}: a_{4}\right) \in \mathbb{P}^{3}(\mathbb{K})$, we represent by $\operatorname{mult}(P, \mathcal{S})$ the multiplicity of $P$ on $\mathcal{S}$ (see [2] p. 258); note that, if $a_{i} \neq 0$, then $\operatorname{mult}(P, \mathcal{S})=\operatorname{mult}\left(\left(\frac{a_{j}}{a_{i}}, \frac{a_{k}}{a_{i}}, \frac{a_{\ell}}{a_{i}}\right), \mathcal{S}_{i}\right)$, where $j<k<\ell$ and $i \notin\{j, k, \ell\}$. 


\section{General STRATEGy AND IDEAS}

We briefly describe here the ideas of our strategy and ideas. The precise details on how to execute them will be presented in subsequent sections. The main steps in our strategy are as follows; we recall that our goal is to decompose $\mathbb{P}^{2}(\mathbb{K})$ such that for $\left(s_{0}: t_{0}: v_{0}\right) \in \mathbb{P}^{2}(\mathbb{K})$ we know whether $\mathcal{P}\left(s_{0}, t_{0}, v_{0}\right)$ is singular or simple in $\mathcal{S}$, and if it is singular we also want to determine its multiplicity. For this purpose, we will denote by $\Delta_{i}$ the algebraic set defined, over $\mathbb{K}$, by the least common multiple of the denominators of $\mathcal{P}_{i}(s, t)$.

[Basic Tool] The basic tool is a formula for computing the multiplicity of an affine point in $\mathbb{K}^{3}$ w.r.t. a rational affine surface, given by means of a rational parametrization (see Section 3 for further details). The formula is based on the computation of the degree of two rational maps, one depending on the point under analysis and the other the map associated to the given parametrization.

Intuitive Idea: Let us assume that we know the defining polynomial $f(x, y, z)$ of the surface, then the multiplicity of the origin is given by the difference of the degree of the homogenization $F(x, y, z, w)$ of $f(x, y, z)$ minus the degree of $F$ w.r.t. $w$. On the other hand, if one is working with parametrizations (which is our case) this computation can be reduced to the computation of two different partial degrees (i.e. degrees w.r.t. a fixed variable), and this can be done, using generalized resultants (see Section 4 in [8]) to count the elements in generic fibers. If the point is not the origin a suitable translation solves the problem. For details see Section 3 .

[Basic Analysis] Once the multiplicity formula has been developed we analyze its behavior, under specialization, when applied to a parametric (rational) point. Indeed, the parametric point is taken as the original rational parametrization of the surface but introducing two new parameters. So, the full analysis is done carrying out 4 parameters: two are representing the surface and the two others are varying and representing particular points on the surface, all through the given parametrization.

Intuitive Idea: Since the formula is, ultimately, based on general resultants, the analysis essentially imply to study how the subsequent gcds and resultants behave under specialization.

(1) [Affine Points] First we analyze the parameter values of the form $\left(s_{0}: t_{0}: 1\right)$. For that, we apply the method, derived from the basic analysis, to study under specializations the multiplicity of $\mathcal{P}_{4}(s, t)$ and we treat the problem in $\mathbb{K}^{2}$. At this stage, we will be able to give an answer for $\mathbb{K}^{2} \backslash \Delta_{4}$. Repeating the process for $\Delta_{4}$, with $\mathcal{P}_{3}(s, t)$, and if necessary with $\mathcal{P}_{2}(s, t)$ and $\mathcal{P}_{1}(s, t)$ we will be able to determine the multiplicity for the parameter values in (see Lemma 2.2)

$$
\mathbb{K}^{2} \backslash \bigcap_{i=1}^{4} \Delta_{i}=\mathbb{K}^{2} \backslash \mathfrak{B}_{a}
$$

Intuitive Idea: We apply (see basic analysis above) the multiplicity formula for parametric points to the parametric point $\mathcal{P}_{4}\left(s^{*}, t^{*}\right)$, that is a generic point of $\mathcal{S}_{4}$, and where $\mathcal{S}_{4}$ is represented by the parametrization $\mathcal{P}_{4}(s, t)$. This will provide $\operatorname{mult}\left(\left(s_{0}: t_{0}: 1\right)\right)=\operatorname{mult}\left(\mathcal{P}_{4}\left(s_{0}, t_{0}\right), \mathcal{S}_{4}\right)$ for all $\left(s_{0}, t_{0}\right) \in$ 
$\mathbb{K}^{2} \backslash \Delta_{4}$; note that $\mathcal{P}_{4}(s, t)$ is not defined at $\Delta_{4}$ and that generically the multiplicity will be 1 . To deal with the parameter values in $\Delta_{4}$, we apply the generic multiplicity formula again, but now to the points $\mathcal{P}_{3}\left(s^{*}, t^{*}\right)$ where $\left(s^{*}, t^{*}\right) \in \Delta_{4} \backslash \Delta_{3}$, that is a generic point of $\mathcal{S}_{3}$, and where $\mathcal{S}_{3}$ is represented by the parametrization $\mathcal{P}_{3}(s, t)$. If necessary we continue the process using $\mathcal{P}_{2}(s, t)$ and $\mathcal{P}_{1}(s, t)$. At the end, we will have $\operatorname{mult}\left(\left(s_{0}: t_{0}: 1\right)\right)$ for all $\left(s_{0}, t_{0}\right) \in \mathbb{K}^{2} \backslash \mathfrak{B}_{a}$. For details see Section 5 .

(2) [Points at Infinity] First, we analyze the case of $(0: 1: 0)$, checking first whether $(0: 1: 0) \in \mathfrak{B}$. Second, we study the case of the parameter values $(1: \lambda: 0)$. For this purpose, first we find those $\lambda$ values generating base points. Afterwards, we study (under a suitable dehomogenization) the rest of the points.

Intuitive Idea: when the point is over $\mathbb{K}$ we apply the basic tool, and when it is parametric we apply the basic analysis.

\section{The MUltiplicity Formula}

In this section we state a formula for computing the multiplicity of a point $A \in \mathbb{K}^{3}$ w.r.t. an affine rational surface $\mathcal{Z}$ in $\mathbb{K}^{3}$, when a rational parametrization $\mathcal{Q}(s, t)$ (not necessarily proper) is provided. For this purpose, we consider the affine version of the composition of the original parametrization $\mathcal{Q}(s, t)$ with the projection from the point $A$ on $\mathbb{K}^{3}$, (that we denote by $\Phi_{23}(A)$ ), and the formula

$$
\operatorname{deg}(\mathcal{Z})-\operatorname{mult}(A, \mathcal{Z})=\frac{\operatorname{deg}\left(\Phi_{23}(A)\right)}{\operatorname{deg}(\mathcal{Q})}
$$

see also the intuitive idea description in step 1 of the general strategy (see Section 2). By using [6] (Theorem 5 or 6 ), we compute $\operatorname{deg}(\mathcal{Q})$, and the degree of $\Phi_{2,3}(A)$ : $\mathbb{K}^{2} \rightarrow \mathbb{K}^{2}$ is computed using the results in [7] (Theorem 1) or [8] (Theorem 4). Nevertheless, $\operatorname{deg}(\mathcal{Z})$ needs to be determined; recall that we do not have the implicit equation. However, if we would know a point $A_{0} \notin \mathcal{Z}$, then $\operatorname{mult}\left(A_{0}, \mathcal{Z}\right)=0$ and

$$
\operatorname{deg}(\mathcal{Z})=\frac{\operatorname{deg}\left(\Phi_{23}\left(A_{0}\right)\right)}{\operatorname{deg}(\mathcal{Q})} .
$$

Therefore, everything is reduced to map-degree computations. But, how do we compute $A_{0} \notin \mathcal{Z}$ ? The idea is as follows. Let us assume that we have computed the degree $m$ w.r.t. a variable (say $x$ ) of the defining polynomial of $\mathcal{Z}$; this computation, once more, can be done by using generalized resultants, (see Section 4 in [8] ). Then, for almost all lines $\mathcal{L}$ orthogonal to the plane $x=0, \operatorname{Card}(\mathcal{L} \cap \mathcal{Z})=m$. Moreover, since we know a parametrization of $\mathcal{Z}$, namely $\mathcal{Q}(s, t)$, we have a description of $\mathcal{L} \cap \mathcal{Z}$ as intersection of plane curves in the variables $\{s, t\}$. So, one can take orthogonal lines till the expected number of intersections is reached. Once such a line is reached, every point, out of the curves defining $\mathcal{L} \cap \mathcal{Z}$, provides a point that does not belong to $\mathcal{Z}$.

In the following, we recall some of the procedures developed in [6], [7] and [8], and we present our method for determining a point out of the surface without knowing the implicit equation as well as a method for deciding whether a parametrically given surface is a cone (detecting cones will be useful in our analysis). For that 
purpose, throughout this section, $\mathcal{Z} \subset \mathbb{K}^{3}$ is a rational affine surface and

$$
\mathcal{Q}(s, t)=\left(\frac{N_{1}(s, t)}{D_{1}(s, t)}, \frac{N_{2}(s, t)}{D_{2}(s, t)}, \frac{N_{3}(s, t)}{D_{3}(s, t)}\right)
$$

a rational parametrization (in reduced form) of $\mathcal{Z}$; we assume that $\mathcal{Z}$ is not a plane. Moreover, let $f(x, y, z)$ be the defining polynomial of $\mathcal{Z}$ and $F(x, y, z, w)$ its homogenization. For $A=(a, b, c) \in \mathbb{K}^{3}$, we consider the polynomial $g(x, y, z)=$ $f(x+a, y+b, z+c)$, as well as $G(x, y, z, w)=F(x+a w, y+b w, z+c w, w)$. It is clear that

$$
\operatorname{mult}(A, \mathcal{Z})=\operatorname{deg}(G)-\operatorname{deg}_{w}(G) .
$$

On the other hand, note that

$$
\left(\frac{N_{1}(s, t)}{D_{1}(s, t)}-a, \frac{N_{2}(s, t)}{D_{2}(s, t)}-b, \frac{N_{3}(s, t)}{D_{3}(s, t)}-c, 1\right)
$$

parametrizes the projective surface defined by $G$. Therefore, since $\mathcal{Z}$ is not a plane, then $N_{1} / D_{1}-a \neq 0$ and hence

$$
\mathcal{Q}^{*}(s, t)=\left(\frac{N_{2}-b D_{2}}{N_{1}-a D_{1}} \cdot \frac{D_{1}}{D_{2}}, \frac{N_{3}-c D_{3}}{N_{1}-a D_{1}} \cdot \frac{D_{1}}{D_{3}}, \frac{D_{1}}{N_{1}-a D_{1}}\right)
$$

parametrizes the affine surface defined by $G(1, y, z, w)$; note that, since $G$ is homogeneous, $\operatorname{deg}_{w}(G)=\operatorname{deg}_{w}(G(1, y, z, w))$. Let us introduce the following notation

$$
\Phi_{2,3}(A)(s, t)=\left(\frac{N_{2}(s, t)-b D_{2}(s, t)}{N_{1}(s, t)-a D_{1}(s, t)} \cdot \frac{D_{1}(s, t)}{D_{2}(s, t)}, \frac{N_{3}(s, t)-c D_{3}(s, t)}{N_{1}(s, t)-a D_{1}(s, t)} \cdot \frac{D_{1}(s, t)}{D_{3}(s, t)}\right)
$$

and let $\Phi_{2,3}(A): \mathbb{K}^{2} \rightarrow \mathbb{K}^{2}$ be the induced map; note that $\Phi_{2,3}(A)$ is indeed the affine version of the composition of the original parametrization $\mathcal{Q}(s, t)$ with the projection from the point $A$ on $\mathbb{K}^{2}$.

In the following we introduce several rational functions and polynomials, namely $\chi_{i}, \mathfrak{g}_{i}, K, \mathfrak{g}^{A}$. All of them depend on $\mathcal{Q}(s, t)$ and $A$. Nevertheless, since this would not be a source of ambiguity for $\chi_{i}, \mathfrak{g}_{i}$ and $K$, and for the sake of simplicity, we omit this dependency for them. However, for $\mathfrak{g}^{A}$ we keep, in the notation, the dependency w.r.t. the point $A$ and we omit $\mathcal{Q}$ since the parametrization stays the same throughout the whole section.

If for $i=1,2, \quad \chi_{i}(s, t)$ denotes the $i$-component of $\Phi_{2,3}(A)(s, t)$, let $\mathfrak{g}_{i}$ be the polynomial

$$
\mathfrak{g}_{i}\left(s, t, h_{1}, h_{2}\right)=\operatorname{Numer}\left(\chi_{i}(s, t)-\chi_{i}\left(h_{1}, h_{2}\right)\right), \quad i=1,2
$$

where $h_{1}, h_{2}$ are new variables, and let $K\left(s, t, h_{1}, h_{2}\right)=\operatorname{gcd}\left(\mathfrak{g}_{1}, \mathfrak{g}_{2}\right)$ where the gcd is computed in $\mathbb{K}\left[h_{1}, h_{2}\right][s, t]$. That is, the polynomials are bivariate polynomials in $\{s, t\}$ with coefficients in the unique factorization domain $\mathbb{K}\left[h_{1}, h_{2}\right]$ (see e.g. Chapter 1 , Section 14 in [15]), and therefore the gcd is unique up to multiplication by units; so, up to multiplication by non-constant polynomials in $\mathbb{K}\left[h_{1}, h_{2}\right]$. The important property of $K$ is its dependency on $\{s, t\}$. Thus, we introduce the polynomial

$$
\mathfrak{g}^{A}\left(s, t, h_{1}, h_{2}\right)= \begin{cases}K\left(s, t, h_{1}, h_{2}\right) & \text { if } \operatorname{deg}_{\{s, t\}}(K)>0 \\ 1 & \text { if } \operatorname{deg}_{\{s, t\}}(K)=0 .\end{cases}
$$

Remark 3.1. We observe that if $\mathfrak{g}^{A}=1$, then the determinant of the Jacobian of $\Phi_{2,3}(A)(s, t)$ is not identically zero (see the preliminary paragraphs to Theorem 1 in [7]). 
In the following theorem and corollaries we assume that none of the projective curves defined by each of the non-constant polynomials in $\left\{N_{1}, N_{2}, N_{3}, D_{1}, D_{2}, D_{3}\right.$, $\left.N_{2}-b D_{2}, N_{3}-c D_{3}, N_{1}-a D_{1}\right\}$ passes through $(0: 1: 0)$; recall that $A=$ $(a, b, c)$. Note that, if necessary, one can always perform a suitable linear change of coordinates such that the above curves satisfy the assumptions; this is equivalent to performing a suitable linear (polynomial) reparametrization of the original parametrization.

Theorem 3.2 (The general formula). It holds that

(1) $\operatorname{mult}(A, \mathcal{Z})=\operatorname{deg}(\mathcal{Z})$ iff $\mathfrak{g}^{A} \neq 1$.

(2) $\operatorname{mult}(A, \mathcal{Z})<\operatorname{deg}(\mathcal{Z})$ iff $\mathfrak{g}^{A}=1$. Furthermore, if $\mathfrak{g}^{A}=1$, then

$$
\operatorname{deg}(\mathcal{Z})-\operatorname{mult}(A, \mathcal{Z})=\frac{\operatorname{deg}\left(\Phi_{23}(A)\right)}{\operatorname{deg}(\mathcal{Q})},
$$

Proof. (1) By Theorem 4 in [7], $\operatorname{deg}_{w}(G)=0$ iff $\mathfrak{g}^{A} \neq 1$. Hence, $\operatorname{mult}(A, \mathcal{Z})=$ $\operatorname{deg}(\mathcal{Z})$ iff $\mathfrak{g}^{A} \neq 1$.

(2) All hypothesis of Theorem 6 in [8] are satisfied. Therefore, $\operatorname{deg}_{w}(G)=\frac{\operatorname{deg}\left(\Phi_{23}(A)\right)}{\operatorname{deg}\left(\mathcal{Q}^{*}\right)}$, and the result follows by taking into account that $\operatorname{deg}(\mathcal{Q})=\operatorname{deg}\left(\mathcal{Q}^{*}\right)$.

Remark 3.3. Note that:

(1) If $\mathfrak{g}^{A} \neq 1$, by Theorem $3.2(1)$, then $A \in \mathcal{Z}$.

(2) For $A \notin \mathcal{Z}, \operatorname{deg}\left(\Phi_{2,3}(A)\right)$ is invariant, indeed it is $\operatorname{deg}(\mathcal{Z}) \operatorname{deg}(\mathcal{Q})$.

(3) Let $a \neq 0$; similarly for $b$ and $c$. We embed $A$ in $\mathbb{P}^{3}(\mathbb{K})$ as $(a: b: c: 1)$. Let $\overline{\mathcal{Z}}$ be the projective closure of $\mathcal{Z}$, and let $\overline{\mathcal{Z}}_{1}$ the dehomogenization of $\overline{\mathcal{Z}}$ w.r.t. $x_{1}=1$. Let $\mathcal{Q}_{1}$ be the corresponding parametrization of $\overline{\mathcal{Z}}_{1}$ generated by $\mathcal{Q}$, let $\bar{A}=\left(\frac{b}{a}, \frac{c}{a}, \frac{1}{a}\right)$, and let $\bar{\Phi}_{2,3}(\bar{A})$ be the induced rational map obtained from $\mathcal{Q}_{1}$ and $\bar{A}$. Then $\operatorname{deg}(\mathcal{Z})=\operatorname{deg}\left(\overline{\mathcal{Z}}_{1}\right), \operatorname{mult}(A, \mathcal{Z})=$ $\operatorname{mult}\left(\bar{A}, \overline{\mathcal{Z}}_{1}\right)$ and $\operatorname{deg}(\mathcal{Q})=\operatorname{deg}\left(\mathcal{Q}_{1}\right)$. Therefore, $\operatorname{deg}\left(\Phi_{2,3}(A)\right)=\operatorname{deg}\left(\bar{\Phi}_{2,3}(\bar{A})\right)$.

Corollary 3.4. $\mathcal{Z}$ is a cone of vertex $A$ if and only if $\mathfrak{g}^{A} \neq 1$.

Proof. Let $\mathcal{Q}(s, t)=t\left(g_{1}(s), g_{2}(s), g_{3}(s)\right)$ be a rational parametrization of $\mathcal{Z}$ where we assume w.l.o.g. that $A$ is the origin $O$. The defining polynomial $f(x, y, z)$ of $\mathcal{Z}$ is a form of degree $d>1$. Therefore $\operatorname{mult}(O, \mathcal{Z})=d=\operatorname{deg}(\mathcal{Z})$ and by Theorem 3.2. $\mathfrak{g}^{O} \neq 1$. Conversely, if $\mathfrak{g}^{A} \neq 1$, then $\operatorname{deg}_{w}(G)=0$ (see Theorem 4 in [7]). Thus, $G(x, y, z, w)=g(x, y, z)$ is an irreducible form of degree $d>1$. Let us see that $\{g(x, y, z)=0\} \cap\{x=1\}$ defines a rational plane curve $\mathcal{D}$ of degree $d$. Since $g$ is a form and $\mathcal{Z}$ is not a plane, $\mathcal{D}$ is a curve of degree $d$. Moreover, since $\mathcal{Z}$ is a surface, $\Phi_{2,3}(A)(s, t)$ is not constant and parametrizes the surface defined by $g(1, y, z)$. Then substituting either $t$ or $s$ by a suitable constant (say $t$ by $t_{0}$ ), $\mathcal{R}(s):=\left(1, \Phi_{2,3}(A)\left(t_{0}, s\right)\right)$ parametrizes $\mathcal{D}$.

Now, $\mathcal{Q}(s, t)=A+t \mathcal{R}(s)$ defines a cone of vertex $A$ contained in $\mathcal{Z}$. Therefore, since $\mathcal{Z}$ is irreducible, it holds that $\mathcal{Z}$ is the previous cone.

Corollary 3.5. There exists at most one $A \in \mathbb{K}^{3}$ such that $\mathfrak{g}^{A} \neq 1$.

Proof. Otherwise, by Corollary 3.4 , it would be a cone over a line, therefore a plane in contradiction to our general assumption.

Corollary 3.6 (The multiplicity formula). Let $A_{0} \in \mathbb{K}^{3} \backslash \mathcal{Z}$ and let $A \in \mathbb{K}^{3}$. Then 
(1) if $\mathfrak{g}^{A}=1$, then

$$
\operatorname{mult}(A, \mathcal{Z})=\frac{\operatorname{deg}\left(\Phi_{23}\left(A_{0}\right)\right)-\operatorname{deg}\left(\Phi_{23}(A)\right)}{\operatorname{deg}(\mathcal{Q})},
$$

(2) if $\mathfrak{g}^{A} \neq 1$, then

$$
\operatorname{mult}(A, \mathcal{Z})=\frac{\operatorname{deg}\left(\Phi_{23}\left(A_{0}\right)\right)}{\operatorname{deg}(\mathcal{Q})}
$$

Proof. We prove (1); similarly for $(2)$. By Theorem 3.2, one has that $(\operatorname{deg}(\mathcal{Z})-$ $\operatorname{mult}(A, \mathcal{Z})) \operatorname{deg}(\mathcal{Q})=\operatorname{deg}\left(\Phi_{23}(A)\right)$, and $\operatorname{deg}(\mathcal{Z}) \operatorname{deg}(\mathcal{Q})=\operatorname{deg}\left(\Phi_{23}\left(A_{0}\right)\right)$. From here the proof is obvious.

Remark 3.7. Note that

(1) if there exists $A_{0}$ such that $\mathfrak{g}^{A_{0}} \neq 1$ (i.e., $\mathcal{Z}$ is a cone), Theorem 3.2 and Corollary 3.6 provide the degree of the surface.

(2) From Corollary [3.6. one deduces that $\operatorname{deg}\left(\Phi_{23}(A)\right)$ is invariant for all the $A \in \mathbb{K}^{3}$ having the same multiplicity w.r.t. $\mathcal{Z}$.

The next corollary is a direct consequence of Corollary 3.6

Corollary 3.8 (Criterion for simple points). Let $A_{0} \in \mathbb{K}^{3} \backslash \mathcal{Z}$ and let $A \in \mathbb{K}^{3}$. The following statements are equivalent:

(1) $A$ is a simple point of $\mathcal{Z}$.

(2) $\mathfrak{g}^{A}=1$ and $\operatorname{deg}\left(\Phi_{2,3}\left(A_{0}\right)\right)-\operatorname{deg}\left(\Phi_{23}(A)\right)=\operatorname{deg}(\mathcal{Q})$.

Proof. By Theorem 3.2, $(\operatorname{deg}(\mathcal{Z})-1) \operatorname{deg}(\mathcal{Q})=\operatorname{deg}\left(\Phi_{2,3}(A)\right)$, and $\operatorname{deg}(\mathcal{Z}) \operatorname{deg}(\mathcal{Q})=$ $\operatorname{deg}\left(\Phi_{2,3}\left(A_{0}\right)\right)$. From here the proof is obvious.

In the last part of this section we deal with the algorithmic outline of the ideas described before. On one hand, we observe that because of Corollaries 3.4, 3.5, and 3.6. it would help our analysis to have an easy way to detect cones. On the other hand, if we know how to compute $\operatorname{deg}(\mathcal{Q}), \operatorname{deg}\left(\Phi_{23}(A)\right)$ for any given $A \in \mathbb{K}^{3}$, and if we know how to compute a point out of the surface (recall that we do not have the implicit equation of $\mathcal{Z}$ ), Corollary 3.6 provides a method for computing the multiplicity of any point in $\mathbb{K}^{3}$, and Corollary 3.8 a method to check whether it is simple on the surface.

\section{CONES DETECTiOn}

For our analysis, it would be helpful to know whether $\mathcal{Q}(s, t)$ is a cone and, if so, to determine its vertex. Let us assume that $\mathcal{Z}$ is a cone. First, we observe that for each $\mathcal{Q}\left(s_{0}, t_{0}\right)$ such that $\left\{\frac{\partial \mathcal{Q}}{\partial s}\left(s_{0}, t_{0}\right), \frac{\partial \mathcal{Q}}{\partial t}\left(s_{0}, t_{0}\right)\right\}$ are linearly independent, the tangent plane to $\mathcal{Z}$ at $\mathcal{Q}\left(s_{0}, t_{0}\right)$ intersects $\mathcal{Z}$ in a line passing through the vertex of the cone; we refer to such points as $\mathcal{Q}$-regular. Our second remark is that the intersection of all tangent planes to $\mathcal{Z}$ at the $\mathcal{Q}$-regular points is indeed the vertex; recall that $\mathcal{Z}$ is not a plane. This is established in the next lemma.

Lemma 3.9. Let $\mathcal{Z}$ be a cone different from a plane. The intersection of all tangent planes to $\mathcal{Z}$ at the $\mathcal{Q}$-regular points is the vertex.

Proof. Since $\mathcal{Z}$ is not a plane, the intersection cannot be a plane. Moreover, the vertex is on all the tangent planes, so it only remains to prove that the intersection is not a line. We assume w.l.o.g. that the vertex is the origin. Then, after a suitable 
birational transformation $\mathcal{Z}$ can be parametrized as $\left(t p_{1}(s), t p_{2}(s), t\right)$ where $p_{1}, p_{2}$ are rational functions, not both constant. Let us assume that the intersection is a line and let $v=(\alpha, \beta, \gamma)$ be its direction vector. Then, $\left\{v,\left(p_{1}(s), p_{2}(s), 1\right),\left(t p_{1}^{\prime}(s)\right.\right.$, $\left.\left.t p_{2}^{\prime}(s), 0\right)\right\}$ are linearly dependent. Thus, $\left\{v,\left(p_{1}(s), p_{2}(s), 1\right),\left(p_{1}^{\prime}(s), p_{2}^{\prime}(s), 0\right)\right\}$ are linearly dependent. Note that $\gamma \neq 0$ because $\mathcal{Z}$ is not a plane. Therefore,

$$
\left(\frac{\alpha}{\gamma}-p_{1}(s)\right) p_{2}^{\prime}(s)=\left(\frac{\beta}{\gamma}-p_{2}(s)\right) p_{1}^{\prime}(s) .
$$

This implies that infinity tangents to the rational curve $\left(p_{1}(s), p_{2}(s)\right)$ pass through the point $\left(\frac{\alpha}{\gamma}, \frac{\beta}{\gamma}\right)$. So, $\left(p_{1}(s), p_{2}(s)\right)$ is a line, and $\mathcal{Z}$ a plane, which is a contradiction.

The next theorem characterizes conical surfaces.

Theorem 3.10. Let $\mathcal{Z}$ not be a plane. $\mathcal{Z}$ is a cone if and only if all tangent planes to $\mathcal{Z}$ at the $\mathcal{Q}$-regular points intersect exactly in one point; in this case, the intersection point is the vertex of the cone.

Proof. The left-right implication was proved in Lemma 3.9. So, let us assume that all tangent planes to $\mathcal{Z}$ at the $\mathcal{Q}$-regular points intersect exactly in one point, that w.l.o.g. we assume is the origin. Let $F(x, y, z)$ be the defining polynomial of $\mathcal{Z}$. Then, for almost all $(s, t)$ values it holds that

$$
\nabla(F)(\mathcal{Q}(s, t)) \cdot(\overline{0}-\mathcal{Q}(s, t))=0 .
$$

Let $G(x, y, z)=x \frac{\partial F}{\partial x}(x, y, z)+y \frac{\partial F}{\partial y}(x, y, z)+z \frac{\partial F}{\partial z}(x, y, z)$; clearly $G$ is not the zero polynomial. Since $F$ is irreducible and $\operatorname{deg}(F)=\operatorname{deg}(G)$, one has that $F=\alpha G$ for some $\alpha \in \mathbb{K}$. On the other hand, let $F$ be expressed as $F=\sum_{i=k}^{n} F_{i}$, where $F_{i}$ is homogenous of degree $i$ and $k \leq n$. Then, $G=\sum_{i=k}^{n}\left(x \frac{\partial F_{i}}{\partial x}+y \frac{\partial \bar{F}_{i}}{\partial y}+z \frac{\partial F_{i}}{\partial z}\right)$. Thus, by Euler's formula, $F_{i}=\alpha\left(x \frac{\partial F_{i}}{\partial x}+y \frac{\partial F_{i}}{\partial y}+z \frac{\partial F_{i}}{\partial z}\right)=\alpha i F_{i}$, for $k \leq i \leq n$. Therefore, $\alpha=1 / n$ and $k=n$. So, $F$ is homogeneous and defines a conical surface.

From the previous results, one derives the following algorithm.

\section{Method 1: Checking cones}

[Step 1] Take parameter values until getting three $\mathcal{Q}$-regular points not on the same line; say $P_{i}=\mathcal{Q}\left(s_{i}, t_{i}\right), i=1,2,3$.

[Step 2] For $i=1,2,3$, compute the tangent plane $H_{i}$ to $\mathcal{Z}$ at $P_{i}$.

[Step 3] If $H_{1} \cap H_{2} \cap H_{3}=\emptyset$, return that $\mathcal{Z}$ is not a cone.

[Step 4] Compute the implicit equation $\Delta(s, t, x, y, z)$ of the tangent plane to $\mathcal{Z}$ at the generic point $\mathcal{Q}(s, t)$.

[Step 5] If $H_{1} \cap H_{2} \cap H_{3}=\{Q\}$ and $\Delta(s, t, Q)$ is identically zero, return that $\mathcal{Z}$ is a cone of vertex $Q$ else return that $\mathcal{Z}$ is not a cone.

[Step 6] If $H_{1} \cap H_{2} \cap H_{3}$ is a line, say parametrized as $L(\lambda)$, and $\Delta(s, t, L(\lambda))$ is identically zero then return $\mathcal{Z}$ is not a cone else

[Step 6.1.] Take $\left(s_{4}, t_{4}\right)$ such that $P_{4}:=\mathcal{Q}\left(s_{4}, t_{4}\right)$ is $\mathcal{Q}$-regular and $\Delta\left(s_{4}, t_{4}, L(\lambda)\right)$ is well defined and non-zero.

[Step 6.2.] Compute the tangent plane $H_{4}$ to $\mathcal{Z}$ at $P_{4}$.

[Step 6.3.] If $H_{1} \cap \cdots \cap H_{4}=\emptyset$, return that $\mathcal{Z}$ is not a cone.

[Step 6.4.] If $H_{1} \cap \cdots \cap H_{4}=\left\{Q^{\prime}\right\}$ and $\Delta\left(s, t, Q^{\prime}\right)$ is identically zero, return $\mathcal{Z}$ is a cone of vertex $Q^{\prime}$ else return that $\mathcal{Z}$ is not a cone. 
[Step 7] If $H_{1} \cap H_{2} \cap H_{3}$ is a plane, say parametrized as $L(\lambda, \mu)$, and $\Delta(s, t, L(\lambda, \mu))$ is identically zero, then return $\mathcal{Z}$ is not a cone else

[Step 7.1.] Take $\left(s_{4}, t_{4}\right)$ such that $P_{4}:=\mathcal{Q}\left(s_{4}, t_{4}\right)$ is $\mathcal{Q}$-regular and $\Delta\left(s_{4}, t_{4}, L(\lambda, \mu)\right)$ is well defined and non-zero.

[Step 7.2.] Compute the tangent plane $H_{5}$ to $\mathcal{Z}$ at $P_{4}$.

[Step 7.3.] If $H_{1} \cap \cdots \cap H_{4}=\emptyset$, return that $\mathcal{Z}$ is not a cone.

[Step 7.4.] If $H_{1} \cap \cdots \cap H_{4}=\left\{Q^{\prime}\right\}$ and $\Delta\left(s, t, Q^{\prime}\right)$ is identically zero, return $\mathcal{Z}$ is a cone of vertex $Q^{\prime}$ else return that $\mathcal{Z}$ is not a cone.

[Step 7.5.] If $H_{1} \cap \cdots \cap H_{4}$ is a line go to Step 6 .

Remark 3.11. Note that, in general, if the parameter values are taken randomly, the algorithm will not need to execute Steps 6 and 7.

\section{Computation of $\operatorname{deg}(\mathcal{Q})$ AND OF $\operatorname{deg}\left(\Phi_{23}(A)\right)$}

We note that $\operatorname{deg}(\mathcal{Q})$ is the index of improperness of $\mathcal{Q}(s, t)$; if $\mathcal{Q}(s, t)$ is proper, then this index is 1 . Therefore, once the parametrization is given, $\operatorname{deg}(\mathcal{Q})$ is fixed. However, $\operatorname{deg}\left(\Phi_{23}(A)\right)$ will vary depending on $A$. Both quantities can be derived by applying elimination theory techniques such as Gröbner basis. Indeed, they can be computed by means of resultants as shown in [6] (Theorem 5 and Theorem 6) without determining the implicit equation of the surface.

In the following we recall (as a recipe) how to compute $\operatorname{deg}(\mathcal{Q})$ and $\operatorname{deg}\left(\Phi_{23}(A)\right)$. In order to compute the degree of a map, we count the number of points in a generic fiber. With our geometric assumptions, this can be computed using resultant computations. This is done in previous papers, but for completeness we recall them here. For further details, in order to compute $\operatorname{deg}(\mathcal{Q})$ we refer to [6] (see Theorems 5 and 6), and to compute $\operatorname{deg}\left(\Phi_{23}(A)\right)$, we refer to [7] (Theorem 1) or [8] (see Theorem 4). In addition, we deduce a method for determining a point out of the surface.

\section{Method 2: Computation of $\operatorname{deg}(\mathcal{Q})$}

[Step 0] Check the global hypotheses:

[Step 0.1] If any of the projective curves defined by the each of the nonconstant polynomials in $\left\{N_{1}, N_{2}, N_{3}, D_{1}, D_{2}, D_{3}\right\}$ passes through $(0: 1: 0)$, apply a suitable (polynomial) linear reparametrization to $\mathcal{Q}(s, t)$; i.e., apply a linear change of coordinates in $\{s, t\}$.

[Step 0.2] If the determinant of the Jacobian of $\left(\frac{N_{2}}{D_{2}}, \frac{N_{3}}{D_{3}}\right)$ is identically zero, apply a suitable linear change of coordinates in $\mathbb{K}^{3}$; namely, exchange suitably the affine coordinates in $\mathbb{K}^{3}$.

[Step 1] For $i=1,2,3$, compute $G_{i}\left(s, t, h_{1}, h_{2}\right)=\operatorname{Numer}\left(\frac{N_{i}(s, t)}{D_{i}(s, t)}-\frac{N_{i}\left(h_{1}, h_{2}\right)}{D_{i}\left(h_{1}, h_{2}\right)}\right)$.

[Step 2] Determine $R\left(s, h_{1}, h_{2}, X\right)=\operatorname{Res}_{t}\left(G_{1}, G_{2}+X G_{3}\right)$ where $X$ is a new variable. [Step 3] Compute $\left.S\left(s, h_{1}, h_{2}\right)=\operatorname{PrimPart}_{\left\{h_{1}, h_{2}\right\}}\left(\operatorname{Content}_{X}(R)\right)\right)$.

[Step 4] $\operatorname{deg}(\mathcal{Q})=\operatorname{deg}_{s}(S)$.

We observe that by Theorem [3.2, if $A_{0} \notin \mathcal{Z}$, then $\mathfrak{g}^{A_{0}}=1$. Therefore, by Corollary [3.6, we only need to compute $\operatorname{deg}\left(\Phi_{23}(A)\right)$ for those $A$ such that $\mathfrak{g}^{A}=1$; in particular when $A=A_{0}$. Thus, in the following we assume that $A=(a, b, c)$ is such that $\mathfrak{g}^{A}=1$.

In addition, since $\mathfrak{g}^{A}=1$, by Remark 3.1, the determinant of the Jacobian of $\Phi_{2,3}(A)(s, t)$ does not vanish. Therefore, $\Phi_{2,3}(A)\left(\mathbb{K}^{2}\right)$ is dense in $\mathbb{K}^{2}$. So, we can 
compute the degree by taking a generic element $\left(\mathrm{X}_{1}, \mathrm{X}_{2}\right) \in \mathbb{K}^{2}$ as is done in [8] (see Theorem 2). More precisely, we have the following method.

\section{Method 3: Computation of $\operatorname{deg}\left(\Phi_{23}(A)\right)$}

[Step 0] Check the global hypotheses: if any of the projective curves defined by each of the non-constant polynomials in $\left\{N_{1}-a D_{1}, N_{2}-b D_{2}, N_{3}-c D_{3}, N_{1}, N_{2}, N_{3}\right.$, $\left.D_{1}, D_{2}, D_{3}\right\}$ passes through $(0: 1: 0)$, apply a suitable (polynomial) linear reparametrization to $\mathcal{Q}(s, t)$; i.e., apply a linear change of coordinates in $\{s, t\}$.

[Step 1] We take the components $\chi_{i}(s, t)$ of $\Phi_{2,3}(A)(s, t):=\left(\chi_{1}(s, t), \chi_{2}(s, t)\right)$.

[Step 2] For $i=1,2$, let $H_{i}\left(s, t, \mathrm{X}_{i}\right)=\operatorname{Numer}\left(\chi_{i}(s, t)-\mathrm{X}_{i}\right) \in \mathbb{K}\left[s, t, \mathrm{X}_{i}\right]$.

[Step 3] $R\left(s, \mathrm{X}_{1}, \mathrm{X}_{2}\right)=\operatorname{Res}_{t}\left(H_{1}, H_{2}\right) \in \mathbb{K}\left[s, \mathrm{X}_{1}, \mathrm{X}_{2}\right]$.

[Step 4] $\operatorname{deg}\left(\Phi_{2,3}(A)\right)=\operatorname{deg}_{s}\left(\operatorname{PrimPart}_{\left\{\mathrm{X}_{1}, \mathrm{X}_{2}\right\}}(R)\right)$.

\section{Computation of a point $A_{0}$ out of $\mathcal{Z}$}

For our reasoning we need to know the degree with respect to one of the variables of the defining polynomial of $\mathcal{Z}$; i.e., its partial degree. Say that $m$ is the degree of the defining polynomial of $\mathcal{Z}$ w.r.t. the variable $x$ (below we show how to compute $m$ ). This means that for almost all affine lines $\mathcal{L}$ of the type $\{y=\lambda, z=\mu\}$ (recall that $\mathcal{Z}$ is not a plane) it holds that $\operatorname{Card}(\mathcal{L} \cap \mathcal{Z})=m$. Then, the idea is as follows. We take values for $(\lambda, \mu)$ till the number of different points on $\mathcal{Z}$ generated by $\mathcal{Q}(s, t)$ is $m$. Note that for a fixed $(\lambda, \mu)$, these points are:

$$
\mathcal{W}(\lambda, \mu)=\left\{\begin{array}{l|l}
\left(\frac{N_{1}(s, t)}{D_{1}(s, t)}, \lambda, \mu\right) \mid \begin{array}{l}
D_{2}(s, t) \lambda-N_{2}(s, t)=0 \\
D_{3}(s, t) \mu-N_{3}(s, t)=0 \\
\operatorname{lcm}\left(D_{1}, D_{2}, D_{3}\right)(s, t) \neq 0
\end{array}
\end{array}\right.
$$

Once we have found a suitable $(\lambda, \mu)$, every point $(\alpha, \lambda, \mu) \notin \mathcal{W}(\lambda, \mu)$ is not on $\mathcal{Z}$. We finish this section showing how to compute $m$ (see details in Theorem 6 in [8]).

Method 4: Computation of the degree w.r.t. $x$

[Step 1] Apply Method 2 to compute $\operatorname{deg}(\mathcal{Q})$.

[Step 2] Let, for $i=2,3, G_{i}\left(s, t, h_{1}, h_{2}\right)$ as in Step 1 of Method 2.

[Step 3] Return $\frac{1}{\operatorname{deg}(\mathcal{Q})} \operatorname{deg}_{s}\left(\operatorname{PrimPart}_{\left\{h_{1}, h_{2}\right\}}\left(\operatorname{Res}_{t}\left(G_{2}\left(s, t, h_{1}, h_{2}\right), G_{3}\left(s, t, h_{1}, h_{2}\right)\right)\right)\right.$.

Remark 3.12. Note that the polynomials $G_{i}$ are obtained in Step 1 of Method 2, and therefore it might happen that Step 0 of Method 2 was required. In that case, we would have performed a linear change in the parameters $\{s, t\}$, and/or an affine linear change of coordinates $\{x, y, z\}$ consisting in a permutation of variables. The first situation does not affect to the partial degree of the polynomial. However, the second can. Nevertheless, if this is the case, we only need to work with the new variable (the one exchanged with $x$ ) and the corresponding lines perpendicular to its corresponding coordinate plane.

Method 5: Computation of $A_{0} \in \mathbb{K}^{3} \backslash \mathcal{Z}$

[Step 1] Apply Method 4 to compute the degree $m$ of the defining polynomial of $\mathcal{Z}$ w.r.t. $x$.

[Step 2] Give values to $(\lambda, \mu) \in \mathbb{K}^{2}$ till $\operatorname{Card}(\mathcal{W}(\lambda, \mu))=m$, then take $A_{0}:=$ $(\alpha, \lambda, \mu) \in \mathbb{K}^{3} \backslash \mathcal{W}(\lambda, \mu)$. 


\section{Computation of $\operatorname{mult}(A, \mathcal{Z})$}

We finish the section, putting together all the previous ideas for computing the multiplicity of $A \in \mathbb{K}^{3}$ w.r.t. to the rational affine surface $\mathcal{Z}$, parametrized by $\mathcal{Q}(s, t)$.

Method 6: Computation of $\operatorname{mult}(A, \mathcal{Z})$

[Step 1] Apply Method 5 to find a point $A_{0} \notin \mathcal{Z}$.

[Step 2] Compute $\mathfrak{g}^{A}$.

[Step 3] If $\mathfrak{g}^{A} \neq 1$, then

[Step 3.1] Apply Method 3 to compute $n_{1}:=\operatorname{deg}\left(\Phi_{2,3}\left(A_{0}\right)\right)$.

[Step 3.2] Apply Method 2 to compute $n_{2}:=\operatorname{deg}(\mathcal{Q})$.

[Step 3.3] Return $\frac{n_{1}}{n_{2}}$.

[Step 4] If $\mathfrak{g}^{A}=1$, then

[Step 4.1] Apply Method 3 to compute $m_{1}:=\operatorname{deg}\left(\Phi_{2,3}(A)\right)$ and

$n_{1}:=\operatorname{deg}\left(\Phi_{2,3}\left(A_{0}\right)\right)$.

[Step 4.2] Apply Method 2 to compute $n_{2}:=\operatorname{deg}(\mathcal{Q})$.

[Step 4.3] Return $\frac{n_{1}-m_{1}}{n_{2}}$.

\section{The MUltiplicity ALgORIthm UNDER SPECIALIZATION}

Let $\mathcal{Q}(s, t)$ and $\mathcal{Z}$ be as in Section 3 . For our purposes, we need to analyze how Method 6 works, under specialization, when applied to a generic point on $\mathcal{Z}$. So, we take $\mathcal{A}\left(s^{*}, t^{*}\right)=\mathcal{Q}\left(s^{*}, t^{*}\right)$ as generic point and we analyze $\operatorname{mult}\left(\mathcal{A}\left(s^{*}, t^{*}\right), \mathcal{Z}\right)$, depending on the values taken by $\left(s^{*}, t^{*}\right)$; note that we need to introduce new parameters $\left(s^{*}, t^{*}\right)$, because in the execution of Method $6, \mathcal{Q}(s, t)$ will represent the surface while $\mathcal{A}\left(s^{*}, t^{*}\right)$ will represent the generic point, and hence the specialization will be considered on $\left(s^{*}, t^{*}\right)$. In addition, we will also study the particular case when the point on the surface does not represent all the surface but only a curve on the surface.

Let $\Delta_{\mathcal{Q}}$ be the algebraic set defined by $\operatorname{lcm}\left(D_{1}, D_{2}, D_{3}\right)$ over $\mathbb{K}$. Then $\mathcal{A}\left(s^{*}, t^{*}\right)$ is well defined in $\Lambda:=\mathbb{K}^{2} \backslash \Delta_{\mathcal{Q}}$. Our goal is to decompose $\Lambda$ as

$$
\Lambda=\bigcup_{k=1}^{\ell} \mathfrak{F}_{k}
$$

such that if $\left(s_{0}, t_{0}\right) \in \mathfrak{F}_{k}$, then $\mathcal{A}\left(s_{0}, t_{0}\right)$ is a point of $\mathcal{Z}$ of multiplicity $k$. For this purpose, we analyze how the steps of Method 6 behave under specialization. Generically, that is, for an open subset of $\Lambda$, the multiplicity will be 1 . We will collect the exceptional situations, i.e. those parameter values where the multiplicity might be bigger than 1 , in a subset $\Omega$ to later analyze the multiplicities in $\Omega \backslash \Delta_{\mathcal{Q}}$.

\section{DETERMination OF $\Omega$}

$\Omega$ is computed as a union of finitely many closed sets $\Omega_{0}, \ldots, \Omega_{4}$. Let us assume that we have already computed a point $A_{0} \in \mathbb{K}^{3} \backslash \mathcal{Z}$ (see Method 5 in Section 3) as well as $\operatorname{deg}\left(\Phi_{2,3}\left(A_{0}\right)\right)$ and $\operatorname{deg}(\mathcal{Q})$ (see Methods 2 and 3 in Section 3); therefore, Step 1 of Method 6 is already executed. To perform Step 2 in Method 6, let $\Phi_{2,3}(\mathcal{A})(s, t)$ be expressed as $\Phi_{2,3}(\mathcal{A})(s, t)=\left(\chi_{1}\left(s, t, s^{*}, t^{*}\right), \chi_{2}\left(s, t, s^{*}, t^{*}\right)\right)$ where

$$
\chi_{1}\left(s, t, s^{*}, t^{*}\right)=\frac{D_{2}\left(s^{*}, t^{*}\right) N_{2}(s, t)-N_{2}\left(s^{*}, t^{*}\right) D_{2}(s, t)}{D_{1}\left(s^{*}, t^{*}\right) N_{1}(s, t)-N_{1}\left(s^{*}, t^{*}\right) D_{1}(s, t)} \cdot \frac{D_{1}(s, t)}{D_{2}(s, t)} \cdot \frac{D_{1}\left(s^{*}, t^{*}\right)}{D_{2}\left(s^{*}, t^{*}\right)},
$$




$$
\chi_{2}\left(s, t, s^{*}, t^{*}\right)=\frac{D_{3}\left(s^{*}, t^{*}\right) N_{3}(s, t)-N_{3}\left(s^{*}, t^{*}\right) D_{3}(s, t)}{D_{1}\left(s^{*}, t^{*}\right) N_{1}(s, t)-N_{1}\left(s^{*}, t^{*}\right) D_{1}(s, t)} \cdot \frac{D_{1}(s, t)}{D_{3}(s, t)} \cdot \frac{D_{1}\left(s^{*}, t^{*}\right)}{D_{3}\left(s^{*}, t^{*}\right)} .
$$

Note that, since $s, t, s^{*}, t^{*}$ are independent variables, and since $\mathcal{Z}$ is not a plane, the above rational functions are well-defined. Moreover, for every particular value $\left(s_{0}, t_{0}\right) \in \Lambda$ of $\left(s^{*}, t^{*}\right)$ the specialization of the rational functions are also welldefined. In addition, since in the following there is no ambiguity on the dependency of $\mathfrak{g}^{\mathcal{A}}$ on $\mathcal{A}$, we simplify the notation writing $\mathfrak{g}$ instead of $\mathfrak{g}^{\mathcal{A}}$.

In this situation, we assume that we have also applied Method 1 to detect whether $\mathcal{Z}$ is a cone. Then, we define $\Omega_{0}$ as the empty set if $\mathcal{Z}$ is not a cone and as $\mathcal{Q}^{-1}(V)$ if $\mathcal{Z}$ is a cone of vertex $V$. Therefore, because of Corollary 3.4, we can assume in the sequel that $\mathfrak{g}=1$. Thus, we skip Steps 2 and 3 of Method 6 . Now, we proceed with Step 4 of Method 6. We have assumed that $n_{1}$ and $n_{2}$ (in Step 4 of Method $6)$ have been already computed. So, it only remains to analyze the determination of $m_{1}:=\operatorname{deg}\left(\Phi_{2,3}(\mathcal{A})\right)$. Therefore, we apply Method 3 to $\Phi_{2,3}(\mathcal{A})(s, t)$.

We assume that none of the projective curves defined by the non-constant polynomials in $\left\{N_{1}, N_{2}, N_{3}, D_{1}, D_{2}, D_{3}\right\}$ passes through $(0: 1: 0)$. If this is not the case, we perform a suitable affine change of coordinates in $\{s, t\}$. Note that, in this situation, $\Phi_{2,3}(\mathcal{A})(s, t)$ satisfies the conditions in Step 0 of Method 3, seeing the projective curves in $\mathbb{P}^{2}\left(\overline{\mathbb{K}\left(s^{*}, t^{*}\right)}\right)$ where $\overline{\mathbb{K}\left(s^{*}, t^{*}\right)}$ is the algebraic closure of $\mathbb{K}\left(s^{*}, t^{*}\right)$. However, it might happen for some particular values of $\left\{s^{*}, t^{*}\right\}$ the condition fails. In order to control this, we introduce the following set $\mathcal{N}_{\infty}$. We take the homogenization (in the variables $\{s, t\}$ ) of the numerators and denominators of $\chi_{i}\left(s, t, s^{*}, t^{*}\right)$, and we substitute them in $(0: 1: 0)$. Observe that, as remarked above, the resulting polynomials are not identically zero. Now, $\mathcal{N}_{\infty}$ is the union of the zero sets in $\mathbb{K}^{2}$ of these polynomials.

In Step 1 of Method 3, we take $\chi_{i}\left(s, t, s^{*}, t^{*}\right), i=1,2$, and in Step 2 of Method 3 , we compute

$$
H_{i}\left(s, t, \mathrm{X}_{i}, s^{*}, t^{*}\right)=\operatorname{Numer}\left(\chi_{i}-\mathrm{X}_{i}\right) \in \mathbb{K}\left[s^{*}, t^{*}, \mathrm{X}_{1}, \mathrm{X}_{2}, s\right][t] .
$$

For $i=1,2$, let $M_{i}\left(s, \mathrm{X}_{1}, \mathrm{X}_{2}, s^{*}, t^{*}\right)$ be the leading coefficient of $H_{i}$ w.r.t. $t$. Then, we define $\Omega_{1}$ as the zero set of all coefficients of $M_{1}$ w.r.t. $\left\{s, \mathrm{X}_{1}, \mathrm{X}_{2}\right\}$ union the zero set of all coefficients of $M_{2}$ w.r.t. $\left\{s, \mathrm{X}_{1}, \mathrm{X}_{2}\right\}$ union $\mathcal{N}_{\infty}$.

In Step 3 of Method 3, the resultant polynomial $R$ is computed and we observe that since $\mathfrak{g}=1, R$ is not identically zero. We see $R$ as a polynomial in $\mathbb{K}\left[s^{*}, t^{*}\right]\left[s, \mathrm{X}_{1}, \mathrm{X}_{2}\right]$, and hence we denote it by $R\left(s, \mathrm{X}_{1}, \mathrm{X}_{2}, s^{*}, t^{*}\right)$. Let $W\left(\mathrm{X}_{1}, \mathrm{X}_{2}\right.$, $\left.s^{*}, t^{*}\right)$ be the leading coefficient of $R$ w.r.t. $s$. Then, we define $\Omega_{2}$ as the zero set of all coefficients of $W$ w.r.t. $\left\{\mathrm{X}_{1}, \mathrm{X}_{2}\right\}$.

In Step 4 of Method 3, we first express $R$ as a polynomial in $\left\{\mathrm{X}_{1}, \mathrm{X}_{2}\right\}$ as

$$
R=\sum_{(i, j) \in J} \bar{a}_{i, j}\left(s, s^{*}, t^{*}\right) \mathrm{X}_{1}^{i} \mathrm{X}_{2}^{j},
$$

where we collect the non-zero coefficients of $R$ w.r.t. $\left\{\mathrm{X}_{1}, \mathrm{X}_{2}\right\}$. We want to control the behavior of the primitive part under specializations, which essentially means to control the content. More precisely, let

$$
a\left(s, s^{*}, t^{*}\right)=\operatorname{gcd}\left(\left\{\bar{a}_{i, j} \mid(i, j) \in J\right\}\right)=\operatorname{Content}_{\left\{\mathrm{x}_{1}, \mathrm{X}_{2}\right\}}(R),
$$

and let

$$
a_{i, j}\left(s, s^{*}, t^{*}\right)=\frac{\bar{a}_{i, j}\left(s, s^{*}, t^{*}\right)}{a\left(s, s^{*}, t^{*}\right)} .
$$


Let $N\left(s^{*}, t^{*}\right)$ be the leading coefficient of $a$ w.r.t. $s$. We analyze (under specializations) the gcd of $\left\{\bar{a}_{i, j} \mid(i, j) \in J\right\}$. We distinguish several cases depending on the cardinality of $J$; we observe that $\operatorname{Card}(J) \neq 1$ since $\operatorname{deg}\left(\Phi_{2,3}(\mathcal{A})\right)>0$.

[Case 1] Let $\operatorname{Card}(J)=2$; say $J=\left\{\left(i_{0}, j_{0}\right),\left(i_{1}, j_{1}\right)\right\}$. We apply Lemma 3 in [11] to $\bar{a}_{i_{0}, j_{0}}, \bar{a}_{i_{1}, j_{1}}$, seen as polynomials in $\mathbb{K}\left[s^{*}, t^{*}\right][s]$. Let $L_{0}\left(s^{*}, t^{*}\right)$ be the leading coefficient of $\bar{a}_{i_{0}, j_{0}}$ w.r.t. $s, L_{1}\left(s^{*}, t^{*}\right)$ be the leading coefficient of $\bar{a}_{i_{1}, j_{1}}$ w.r.t. $s$, and let $S\left(s^{*}, t^{*}\right)=\operatorname{Res}_{s}\left(a_{i_{0}, j_{0}}, a_{i_{1}, j_{1}}\right)$. Then, we define $\Omega_{3}$ as the zero set of $\left\{L_{0}, L_{1}\right\}$ union the zero set of $S$, and $\Omega_{4}$ as the zero set of $N$ (see above).

[Case 2] Let $\operatorname{Card}(J)>2$; say $J=\left\{\left(i_{k}, j_{k}\right)\right\}_{k=1, \ldots, \ell}$, with $\ell>2$. We apply Lemma 9 in [6]. For convenience of the reader we recall here the part of that lemma that we will use.

Lemma 4.1 (Lemma 9 in [6]). Let $f_{i} \in \mathbb{K}\left[s^{*}, t^{*}\right][s] \backslash\{0\}, f_{i}=\bar{f}_{i} \cdot \operatorname{gcd}\left(f_{1}, \ldots, f_{m}\right)$, $i=1, \ldots, m$. Let $\left(s_{0}, t_{0}\right) \in \mathbb{K}^{2}$ be such that the leading coefficient of $f_{1}$ w.r.t. $s$ does not vanish at $\left(s_{0}, t_{0}\right)$. If $\operatorname{Res}_{s}\left(\bar{f}_{1}, \bar{f}_{2}+\sum_{i=3}^{m} W_{i-2} \bar{f}_{i}\right)\left(s_{0}, t_{0}\right) \neq 0$, where $W_{j}, j=$ $1, \ldots, m-2$, are new variables, then $\operatorname{gcd}\left(f_{1}, \ldots, f_{m}\right)\left(s_{0}, t_{0}, s\right)=\operatorname{gcd}\left(f_{1}\left(s_{0}, t_{0}, s\right), \ldots\right.$, $\left.f_{m}\left(s_{0}, t_{0}, s\right)\right)$.

Thus, we apply the lemma to $\left\{\bar{a}_{i_{k}, j_{k}}\right\}_{k=1, \ldots, \ell}$ seen as polynomials in $\mathbb{K}\left[s^{*}, t^{*}\right][s]$. Let $L\left(s^{*}, t^{*}\right)$ be the leading coefficient of $\bar{a}_{i_{1}, j_{1}}$ w.r.t. $s$, and let

$$
\bar{S}\left(s^{*}, t^{*}, W_{1}, \ldots, W_{\ell-2}\right)=\operatorname{Res}_{s}\left(a_{i_{1}, j_{1}}, a_{i_{2}, j_{2}}+\sum_{k=3}^{\ell} W_{k-2} a_{i_{k}, j_{k}}\right) .
$$

We define $\Omega_{3}$ as the zero set of all coefficients of $\bar{S}$ w.r.t. $\left\{W_{1}, \ldots, W_{\ell-2}\right\}$, and $\Omega_{4}$ as the zero set of $L$ union the zero set of $N$.

Finally we take $\Omega=\Omega_{0} \cup \cdots \cup \Omega_{4}$.

Note that, since $\mathcal{Z}$ is irreducible, and $\mathcal{A}\left(s^{*}, t^{*}\right)$ is a generic element of $\mathcal{Z}$, we have the following lemma.

Lemma 4.2. Let $\left(s_{0}, t_{0}\right) \in \Lambda$ be such that

$$
\operatorname{deg}\left(\Phi_{2,3}\left(\mathcal{A}\left(s_{0}, t_{0}\right)\right)\right)=\operatorname{deg}_{s}\left(\frac{R\left(s, \mathrm{X}_{1}, \mathrm{X}_{2}, s^{*}, t^{*}\right)}{a\left(s, s^{*}, t^{*}\right)}\right) .
$$

It holds that $\mathcal{A}\left(s_{0}, t_{0}\right)$ is a simple point of $\mathcal{Z}$.

We finish this subsection with the following theorem.

Theorem 4.3. $\forall\left(s_{0}, t_{0}\right) \in \Lambda \backslash \Omega, \mathcal{A}\left(s_{0}, t_{0}\right)$ is a simple point of $\mathcal{Z}$.

Proof. Let $\left(s_{0}, t_{0}\right) \in \Lambda \backslash \Omega$; throughout the proof, we denote $\mathcal{A}\left(s_{0}, t_{0}\right)$ by $\mathcal{A}_{0}$. Since $\left(s_{0}, t_{0}\right) \in \Lambda$, then $\left(s_{0}, t_{0}\right) \notin \Omega_{4}$, and hence $\mathcal{A}_{0}$ is well defined and it is a point on $\mathcal{Z}$. Moreover, $\chi_{i}\left(s_{0}, t_{0}, s, t\right)$ are also well defined. On the other hand, since $\left(s_{0}, t_{0}\right) \notin \Omega_{0}$, then $\mathfrak{g}\left(s, t, h_{1}, h_{2}, s_{0}, t_{0}\right)=1$. Note that since $\left(s_{0}, t_{0}\right) \notin \Omega_{1}$, then $\left(s_{0}, t_{0}\right) \notin \mathcal{N}_{\infty}$ and hence the conditions in Step 0, Method 3, are satisfied. Moreover, neither $M_{1}\left(s, \mathrm{X}_{1}, \mathrm{X}_{2}, s_{0}, t_{0}\right)$ nor $M_{2}\left(s, \mathrm{X}_{1}, \mathrm{X}_{2}, s_{0}, t_{0}\right)$ vanish. Similarly, since $\left(s_{0}, t_{0}\right) \notin \Omega_{2}$, $W\left(\mathrm{X}_{1}, \mathrm{X}_{2}, s_{0}, t_{0}\right)$ does not vanish.

If we are in Case 1 , since $\left(s_{0}, t_{0}\right) \notin \Omega_{3}$ we get that $L_{0}\left(s_{0}, t_{0}\right) \neq 0$ or $L_{1}\left(s_{0}, t_{0}\right) \neq$ 0 , and $S\left(s_{0}, t_{0}\right) \neq 0$. Thus, by Lemma 3 in [11, we get that $a\left(s, s_{0}, t_{0}\right)=$ $\operatorname{gcd}\left(\bar{a}_{i_{0}, j_{0}}\left(s, s_{0}, t_{0}\right), \bar{a}_{i_{1}, j_{1}}\left(s, s_{0}, t_{0}\right)\right)$. Moreover, by well-know properties of resultants, we get that (up to multiplication by a non-zero constant), $R\left(s, \mathrm{X}_{1}, \mathrm{X}_{2}, s_{0}, t_{0}\right)$ $=\operatorname{Res}_{t}\left(H_{1}\left(s, t, \mathrm{X}_{1}, s_{0}, t_{0}\right), H_{2}\left(s, t, \mathrm{X}_{2}, s_{0}, t_{0}\right)\right)$. Furthermore, since $W\left(\mathrm{X}_{1}, \mathrm{X}_{2}, s_{0}, t_{0}\right)$ $\neq 0$ (see above),

$$
\operatorname{deg}_{s}\left(R\left(s, \mathrm{X}_{1}, \mathrm{X}_{2}, s_{0}, t_{0}\right)\right)=\operatorname{deg}_{s}\left(R\left(s, \mathrm{X}_{1}, \mathrm{X}_{2}, s^{*}, t^{*}\right)\right) .
$$


On the other hand, $\left(s_{0}, t_{0}\right) \notin \Omega_{4}$ implies that $\operatorname{deg}_{s}\left(a\left(s, s^{*}, t^{*}\right)\right)=\operatorname{deg}_{s}\left(a\left(s, s_{0}, t_{0}\right)\right)$. Summarizing,

$$
\begin{aligned}
& \operatorname{deg}\left(\Phi_{2,3}\left(\mathcal{A}_{0}\right)\right)=\operatorname{deg}_{s}\left(\operatorname{PrimPart}_{\left\{\mathrm{X}_{1}, \mathrm{X}_{2}\right\}}\left(R\left(s, \mathrm{X}_{1}, \mathrm{X}_{2}, s_{0}, t_{0}\right)\right)\right) \\
= & \operatorname{deg}_{s}\left(\operatorname{PrimPart}_{\left\{\mathrm{X}_{1}, \mathrm{X}_{2}\right\}}\left(R\left(s, \mathrm{X}_{1}, \mathrm{X}_{2}, s^{*}, t^{*}\right)\right)\right)=\operatorname{deg}\left(\Phi_{2,3}(\mathcal{A})\right) .
\end{aligned}
$$

Therefore, by Lemma 4.2, $\mathcal{A}_{0}$ is simple.

If we are in Case 2, since $\left(s_{0}, t_{0}\right) \notin \Omega_{3}$ we get that $\bar{S}\left(s_{0}, t_{0}, W_{1}, \ldots, W_{\ell-2}\right) \neq 0$. Since $\left(s_{0}, t_{0}\right) \notin \Omega_{4}$ we know that $L\left(s_{0}, t_{0}\right) \neq 0$ and $N\left(s_{0}, t_{0}\right) \neq 0$. Thus, by Lemma 4.1. we get that $a\left(s, s_{0}, t_{0}\right)=\operatorname{gcd}\left(\left\{\bar{a}_{i, j}\left(s, s_{0}, t_{0}\right) \mid(i, j) \in J\right\}\right)$. From here the proof follows as in the Case 1.

Observe that, because of Theorem 4.3, $\Lambda \backslash \Omega \subset \mathfrak{F}_{1}$. We finish the subsection, outlining the method for computing $\Omega$.

\section{Method 7: Computation of $\Omega$}

Input: an affine rational surface $\mathcal{Z} \subset \mathbb{K}^{3}$ different to a plane, and a rational parametrization (in reduced form) $\mathcal{Q}(s, t)$ of $\mathcal{Z}$.

Output: the set $\Omega$.

[Step 1] Compute (see Section 5) $\Phi_{2,3}\left(\mathcal{A}\left(s^{*}, t^{*}\right)\right)=\left(\chi_{1}\left(s, t, s^{*}, t^{*}\right), \chi_{2}\left(s, t, s^{*}, t^{*}\right)\right)$.

[Step 2] Computation of $\Omega_{0}$ : Apply Method 1 to $\mathcal{Q}$ to check whether $\mathcal{Z}$ is a cone.

If it is not a cone, then $\Omega_{0}:=\emptyset$ else $\Omega_{0}:=\mathcal{Q}^{-1}(V)$ where $V$ is the vertex of $\mathcal{Z}$.

[Step 3] Computation of $\Omega_{1}$.

[Step 3.1] Homogenize (w.r.t. $\{s, t\}$ ) the numerators and denominators of $\chi_{i}$,

and substitute them in $(0: 1: 0)$. Take $\mathcal{N}_{\infty}$ as the union of the zero sets in $\mathbb{K}^{2}$ of these polynomials.

[Step 3.2] Compute $H_{i}=\operatorname{Numer}\left(\chi_{i}-\mathrm{X}_{i}\right)$. Let $M_{i}$ be the leading coefficient of $H_{i}$ w.r.t. $t$.

[Step 3.3] $\Omega_{1}$ is the zero set of all coefficients of $M_{1}$ w.r.t. $\left\{s, \mathrm{X}_{1}, \mathrm{X}_{2}\right\}$ union the zero set of all coefficients of $M_{2}$ w.r.t. $\left\{s, \mathrm{X}_{1}, \mathrm{X}_{2}\right\}$ union $\mathcal{N}_{\infty}$.

[Step 4] Computation of $\Omega_{2}$.

[Step 4.1] Compute $R:=\operatorname{Res}_{t}\left(H_{1}, H_{2}\right)$ and its leading coefficient $W$ w.r.t. $s$.

[Step 4.2] $\Omega_{2}$ is the zero set of all coefficients of $W$ w.r.t. $\left\{\mathrm{X}_{1}, \mathrm{X}_{2}\right\}$.

[Step 5] Computation of $\Omega_{3}$ and $\Omega_{4}$.

[Step 5.1] Compute the set $\left\{\bar{a}_{i, j} \mid(i, j) \in J\right\}$ of all coefficients of $R$ w.r.t. $\left\{\mathrm{X}_{1}, \mathrm{X}_{2}\right\}$.

[Step 5.2] Compute $a=\operatorname{gcd}\left(\left\{\bar{a}_{i, j} \mid(i, j) \in J\right\}\right)$ and $a_{i, j}=\frac{\bar{a}_{i, j}}{a}$.

[Step 5.3] Determine the leading coefficient $N$ of $a$ w.r.t. $s$.

[Step 5.4] If $\operatorname{Card}(J)=2$ (say $\left.J=\left\{\left(i_{0}, j_{0}\right),\left(i_{1}, j_{1}\right)\right\}\right)$.

[Step 5.4.1] Compute the leading coefficient $L_{j}$ of $a_{i_{j}, j_{j}}$ w.r.t. $s(j=0,1)$

and $S=\operatorname{Res}_{s}\left(a_{i_{0}, j_{0}}, a_{i_{1}, j_{1}}\right)$.

[Step 5.4.2] $\Omega_{3}$ is the zero set of $\left\{L_{0}, L_{1}\right\}$ union the zero set of $S$.

[Step 5.4.3] $\Omega_{4}$ is the zero set of $N$.

[Step 5.5] If $\operatorname{Card}(J)>2\left(\right.$ say $\left.J=\left\{\left(i_{k}, j_{k}\right)\right\}_{k=1, \ldots, \ell}\right)$

[Step 5.5.1] Compute the leading coefficient $L$ of $a_{i_{1}, j_{1}}$ w.r.t. $s$ and $\bar{S}=$

$\operatorname{Res}_{s}\left(a_{i_{1}, j_{1}}, a_{i_{2}, j_{2}}+\sum_{k=3}^{\ell} W_{k-2} a_{i_{k}, j_{k}}\right)$.

[Step 5.5.2] $\Omega_{3}$ is the zero set of all coefficients of $\bar{S}$ w.r.t. $\left\{W_{1}, \ldots, W_{\ell-2}\right\}$.

[Step 5.5.3] $\Omega_{4}$ is the zero set of $L$ union the zero set of $N$.

[Step 6] Return $\Omega:=\Omega_{0} \cup \cdots \cup \Omega_{4}$. 


\section{Computing the Multiplicity in $\Omega \backslash \Delta_{\mathcal{Q}}$}

We decompose $\Omega$ as a union of irreducible closed sets; note that they are of dimension less than or equal to 1 . Let $\mathcal{C}$ be an irreducible curve in $\Omega$. If $\mathcal{C} \subset \Delta_{\mathcal{Q}}$, there is nothing to do. If not, we compute the intersection of $\Delta_{\mathcal{Q}}$ and $\mathcal{C}$ (note that $\Delta_{\mathcal{Q}}$ is empty or a plane curve). This intersection would be either empty or finitely many points. For an open subset of $\mathcal{C}$, the degree of the corresponding map $\Phi_{2,3}$ would be invariant, and hence all points in the open subset would generate points on $\mathcal{S}$ with the same multiplicity. The complementary of this open subset is now either empty or a finite set of points. So, if it is not empty, we apply the formula to each of the finitely many points in the closed set as well as for those points in the zero-dimensional components of $\Omega$.

In order to compute the open subset of $\mathcal{C}$, we do an analogous reasoning as in the previous subsection. One may distinguish two cases:

[General case] We work over the field of rational functions $\mathbb{K}(\mathcal{C})$ of the curve (see [12]). Let $f(s, t)$ be the defining polynomial of $\mathcal{C}$, then $\mathbb{K}(\mathcal{C})$ is the quotient field of $\mathbb{K}[s, t] /(f)$. Then, we apply Method 6 to $\mathcal{A}(\mathbf{s}, \mathbf{t})$, where $\mathbf{s}, \mathbf{t} \in \mathbb{K}(\mathcal{C})$ are the equivalent classes of $s, t$, respectively, i.e., $\mathbf{t}=t+(f)$, and $\mathbf{s}=s+(f)$ with $(f)$ the ideal of $f$. We recall that the arithmetic in the field $\mathbb{K}(\mathcal{C})$ can be executed by using the defining polynomial of $\mathcal{C}$. We observe that all computations can be carried out: we have to compute gcds in $\mathbb{K}(\mathcal{C})\left[h_{1}, h_{2}, s, t\right]$ (which can be performed in the Euclidean domain $\left.\mathbb{K}(\mathcal{C})\left(h_{1}, h_{2}, s\right)[t]\right)$, resultants in the unique factorization domain $\mathbb{K}(\mathcal{C})\left[s, \mathrm{X}_{1}, \mathrm{X}_{2}\right][t]$ and gcds in the Euclidean domain $\mathbb{K}(\mathcal{C})[s]$.

For each 1-dimensional component $\mathcal{C}$ of $\Omega$ we will get an open subset where all points (i.e., parameter values) behave the same; that is all have the same multiplicity. So each of these open subsets will be part of $\mathfrak{F}_{k}$ for some $k$. The complementary of these open sets are either empty or zero-dimensional. So we will have, in the worst case, a set of finitely many parameter values to be classified. For each of them we apply Method 6, and we determine their multiplicity. Finally, they are included in the corresponding $\mathfrak{F}_{k}$.

[Rational case] If $\mathcal{C}$ is rational, we compute a proper normal rational parametrization $\mathcal{M}(\lambda)$ of $\mathcal{C}$ (see [12], Section 6.3). Then, we apply Method 6 to $\mathcal{H}(\lambda):=\mathcal{Q}(\mathcal{M}(\lambda))$; say that $\mathcal{H}(\lambda)$ is expressed as:

$$
\mathcal{H}(\lambda)=\left(\frac{\varphi_{1}(\lambda)}{\phi_{1}(\lambda)}, \frac{\varphi_{2}(\lambda)}{\phi_{2}(\lambda)}, \frac{\varphi_{3}(\lambda)}{\phi_{3}(\lambda)}\right)
$$

where $\operatorname{gcd}\left(\varphi_{i}, \phi_{i}\right)=1, i=1,2,3$. Note that Step 1 as well $n_{1}, n_{2}$ (in Steps 3, 4) were already computed when determining $\Omega$. Let $\mathfrak{g}^{\mathcal{H}(\lambda)}$ be the corresponding gcd generated by the parametric point $\mathcal{H}(\lambda)$ (see Section 3 ): In Step 2, we have to compute and analyze $\mathfrak{g}^{\mathcal{H}(\lambda)}$. We can assume that $\mathfrak{g}^{\mathcal{H}(\lambda)}=1$, since the case $\mathfrak{g}^{\mathcal{H}(\lambda)} \neq 1$ corresponds to the parameter values in $\mathcal{C} \cap \Omega_{0}$, and for these points one applies directly Step 3 in Method 6 to get that the multiplicity is always $\operatorname{deg}(\mathcal{Z})$ (see Theorem 3.2). Thus, we pass to Step 4, and hence it only remains to apply Method 3 to compute $\operatorname{deg}\left(\Phi_{2,3}(\mathcal{H}(\lambda))\right.$ ), where $\lambda$ belongs to a non-empty open subset of $\mathbb{K}$; namely those $\lambda$ such that $\mathcal{M}(\lambda) \in \mathcal{C} \backslash \Omega_{0}$. We observe that all computations can be carried out: we have to compute resultants in the unique factorization domain $\mathbb{K}\left[\lambda, s, \mathrm{X}_{1}, \mathrm{X}_{2}\right][t]$ and gcds in the Euclidean domain $\mathbb{K}(\lambda)[s]$. 
Remark 4.4. Obviously, the genus zero case can be treated as the general case; the fact is that $\mathbb{K}(\mathcal{C})$ turns out to be $\mathbb{K}(\lambda)$. We have not analyzed, from the computational/complexity point of view, which method is better. Using the general case option implies to work with equivalence classes and the length of the integer coefficients in the polynomial representatives tends to grow up. On the other hand, using the rational case option, simplifies, in principle, the arithmetic in the field. However, although the normal parametrization is not hard to achieve, the parametrization may require algebraic extensions of the ground field or, instead, high length coefficients.

We finish this section with the outline of the algorithm that analyzes the multiplicity under specialization.

\section{Method 8: Computation of the multiplicity under specialization} Input:

- An affine rational surface $\mathcal{Z} \subset \mathbb{K}^{3}$ different to a plane.

- A point $A_{0}=(a, b, c) \in \mathbb{K}^{3}$ such that $A_{0} \notin \mathcal{Z}$ (apply Method 5).

- A rational parametrization (in reduced form)

$$
\mathcal{Q}(s, t)=\left(\frac{N_{1}(s, t)}{D_{1}(s, t)}, \frac{N_{2}(s, t)}{D_{2}(s, t)}, \frac{N_{3}(s, t)}{D_{3}(s, t)}\right)
$$

of $\mathcal{Z}$, and such that none of the projective curves defined by each of the nonconstant polynomials in $\left\{N_{1}, N_{2}, N_{3}, D_{1}, D_{2}, D_{3}\right\}$ passes through (0:1:0).

- A set $\Gamma \subseteq \mathbb{K}^{2}$. Set $\Theta$ as $\Theta:=\Gamma \backslash \Delta_{\mathcal{Q}}$, where $\Delta_{\mathcal{Q}}$ is the algebraic set defined over $\mathbb{K}$ by the denominators of $\mathcal{Q}(s, t)$ (that is, $\Delta_{\mathcal{Q}}$ is the algebraic set defined by $\left.\operatorname{lcm}\left(D_{1}, D_{2}, D_{3}\right)\right)$.

- $n_{2}:=\operatorname{deg}(\mathcal{Q})(\operatorname{apply} M e t h o d 2)$ and $n_{1}:=\operatorname{deg}\left(\Phi_{2,3}\left(A_{0}\right)\right)$ (apply Method 3).

Output: A decomposition of $\Theta$ of the form $\bigcup_{k=1}^{\ell} \mathfrak{F}_{k}$, such that if $\left(s_{0}, t_{0}\right) \in \mathfrak{F}_{k}$, then $\mathcal{A}\left(s_{0}, t_{0}\right)$ is a point of $\mathcal{Z}$ of multiplicity $k$.

[Step 0] If $\operatorname{dim}(\Theta)=0$ then apply, to each point in $\Theta$, Method 6 (observe that $A_{0}, n_{1}, n_{2}$ are given) and include the points in the corresponding $\mathfrak{F}_{k}$. If $\operatorname{dim}(\Theta)=1$, take $\Omega$ as the Zariski closure of $\Theta$ and go to Step 4 .

[Step 1] Let $\Omega:=\bigcup_{i=0}^{4} \Omega_{i}$ be the output of the Method 7 applied to $\mathcal{Q}(s, t)$. Include $\Theta \backslash \Omega$ in $\mathfrak{F}_{1}$.

[Step 2] Decompose $\Omega$ into irreducible components.

[Step 3] For each point $A$ at a zero-dimensional component of $\Omega$, if $A \notin \Omega_{4}$, then apply Method 6 to compute $\alpha=\operatorname{mult}(A, \mathcal{Z})$, and include $A$ in $\mathfrak{F}_{\alpha}$.

[Step 4] For each 1-dimensional irreducible component $\mathcal{C}$ of $\Omega$, proceed as we have described (general case) in this section. This will generate an open subset $\mathcal{C}^{*}$ of $\mathcal{C}$ where the multiplicity is invariant and that would be included in the corresponding $\mathfrak{F}_{k}$. For the finitely many points in the $\mathcal{C} \backslash \mathcal{C}^{*}$, proceed as in Step 3. Alternatively, if $\mathcal{C}$ is rational one may proceed as described in the genus zero case.

\section{Computing the $\mathcal{P}$-Singularities}

In this section, we apply the previous results to the resolution of the problem stated in the introduction. We first will deal with the affine singularities and afterwards, we will treat the singularities at infinity. For this purpose, in this section, we work with the projective surface $\mathcal{S}$ and the projective parametrization $\mathcal{P}(\mathfrak{s}, \mathfrak{t}, \mathfrak{v})$ introduced in Section 2. Furthermore, for $i=1,2,3,4$, we consider the affine 
parametrizations $\mathcal{P}_{i}(s, t)$ and the affine surfaces $\mathcal{S}_{i}$ associated to $\mathcal{P}(\mathfrak{s}, \mathfrak{t}, \mathfrak{v})$, as described in Section 2. Moreover, let $\mathfrak{B}, \mathfrak{B}_{a}$ be the set of projective and affine base points of $\mathcal{P}(\mathfrak{s}, \mathfrak{t}, \mathfrak{v})$ (see Section 2), and let $\Delta_{i}$ be the algebraic sets introduced in Lemma 2.2

\section{AfFine $\mathcal{P}$-Singularities}

In this section, we see $\mathbb{K}^{2}$ embedded in $\mathbb{P}^{2}(\mathbb{K})$ by means of the natural map

$$
\mathbf{j}: \mathbb{K}^{2} \rightarrow \mathbb{P}^{2}(\mathbb{K}),\left(s_{0}, t_{0}\right) \mapsto\left(s_{0}: t_{0}: 1\right) .
$$

The basic idea consists in applying Method 8 to a generic point on $\mathcal{S}$. For this purpose, we proceed as follows.

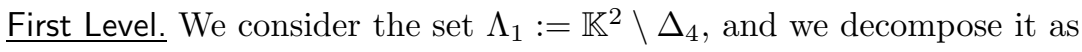

$$
\Lambda_{1}:=\bigcup_{k=1}^{\ell_{1}} \mathfrak{F}_{k}^{1}
$$

such that if $\left(s_{0}, t_{0}\right) \in \mathfrak{F}_{k}^{1}$, then $\mathcal{P}_{4}\left(s_{0}, t_{0}\right)$ is a point of $\mathcal{S}_{4}$ of multiplicity $k$. Second Level. If $\Delta_{4} \backslash \mathfrak{B}_{a} \neq \emptyset$, we consider the set $\Lambda_{2}:=\Delta_{4} \backslash \Delta_{3}$ and we decompose it as

$$
\Lambda_{2}:=\bigcup_{k=1}^{\ell_{2}} \mathfrak{F}_{k}^{2}
$$

such that if $\left(s_{0}, t_{0}\right) \in \mathfrak{F}_{k}^{2}$, then $\mathcal{P}_{3}\left(s_{0}, t_{0}\right)$ is a point of $\mathcal{S}_{3}$ of multiplicity $k$. Third level. If $\Lambda_{2} \backslash \mathfrak{B}_{a} \neq \emptyset$, we consider the set $\Lambda_{3}:=\left(\Delta_{4} \cap \Delta_{3}\right) \backslash \Delta_{2}$ and we decompose it as

$$
\Lambda_{3}=\bigcup_{k=1}^{\ell_{3}} \mathfrak{F}_{k}^{3}
$$

such that if $\left(s_{0}, t_{0}\right) \in \mathfrak{F}_{k}^{3}$, then $\mathcal{P}_{2}\left(s_{0}, t_{0}\right)$ is a point of $\mathcal{S}_{2}$ of multiplicity $k$. Fourth Level. If $\Lambda_{3} \backslash \mathfrak{B}_{a} \neq \emptyset$ we consider the set $\Lambda_{4}:=\left(\Delta_{4} \cap \Delta_{3} \cap \Delta_{2}\right) \backslash \Delta_{1}$ and we decompose it as

$$
\Lambda_{4}=\bigcup_{k=1}^{\ell_{4}} \mathfrak{F}_{k}^{4}
$$

such that if $\left(s_{0}, t_{0}\right) \in \mathfrak{F}_{k}^{4}$, then $\mathcal{P}_{1}\left(s_{0}, t_{0}\right)$ is a point of $\mathcal{S}_{1}$ of multiplicity $k$.

Note that at this point, $\Lambda_{4} \backslash \mathfrak{B}_{a}=\emptyset$. Moreover $\mathbf{j}\left(\bigcup_{i=1}^{4} \mathfrak{F}_{k}^{i}\right) \subset \mathfrak{S}_{k}$ (see the definition of $\mathfrak{S}_{k}$ in the problem statement in Section 11).

In order to deal with the first level, we apply Method 8 to the parametrization $\mathcal{P}_{4}(s, t)$ of the affine surface $\mathcal{S}_{4}$, taking $\mathcal{A}\left(s^{*}, t^{*}\right)=\mathcal{P}_{4}\left(s^{*}, t^{*}\right)$. Let us see how to proceed with the second, third and fourth levels. In level 2 , let $\Delta_{4} \backslash \mathfrak{B}_{a} \neq \emptyset$, then we want to decompose $\Lambda_{2}$ (i.e., $\Delta_{4} \backslash \Delta_{3}$ ). We observe that $\Lambda_{2}$ would be either empty or 1-dimensional; since $\Delta_{i}$ are either empty or plane curves. Clearly, the interesting case is when $\operatorname{dim}\left(\Lambda_{2}\right)=1$. Then, for each irreducible component of $\Lambda_{2}$ we proceed as described in Section 4 Finally, note that the same argument and strategy is valid for the third and the fourth levels. 


\section{$\mathcal{P}$-SINGULARITIES AT INFINITY}

We show how to proceed with Step 2 of our strategy (see Section 2). So, first we analyze whether $A=(0: 1: 0)$ is a $\mathcal{P}$-singularity. For this purpose, we check whether $A \in \mathfrak{B}$. If $A \notin \mathfrak{B}$, then at least one of the polynomials $\mathfrak{p}_{i}$ of $\mathcal{P}(\mathfrak{s}, \mathfrak{t}, \mathfrak{v})$ does not vanish on $A$ (say w.l.o.g. $\mathfrak{p}_{4}$ ). Then, we work with the affine parametrization of $\mathcal{S}_{4}$ obtained by taking $\mathfrak{t}=1$ and dehomogenizing $\mathcal{P}(\mathfrak{s}, \mathfrak{t}, \mathfrak{v})$ w.r.t. to the fourth component. To distinguish this new affine parametrization of $\mathcal{S}_{4}$ from $\mathcal{P}_{4}(s, t)$, we denote it by $\mathcal{P}_{4}^{\mathfrak{t}}(s, t)$. Similarly, if necessary, we introduce $\mathcal{P}_{i}^{\mathfrak{t}}(s, t)$ with $i=1,2,3$. Now, we apply Method 6 to compute

$$
\operatorname{mult}((0: 1: 0))=\operatorname{mult}\left(\mathcal{P}_{4}^{\mathfrak{t}}(0,0), \mathcal{S}_{4}\right)=\operatorname{mult}(\mathcal{P}(0,1,0), \mathcal{S}) .
$$

Now, it only remains to analyze the points in $\mathcal{E}=\left\{\left(1: \lambda_{0}: 0\right) \mid \lambda_{0} \in \mathbb{K}\right\}$. For that, first we determine those points in $\mathcal{E}$ that are base points, namely

$$
\mathcal{E}^{*}=\left\{\left(1: \lambda_{0}: 0\right) \mid \operatorname{gcd}\left(\mathfrak{p}_{1}(1, \lambda, 0), \mathfrak{p}_{2}(1, \lambda, 0), \mathfrak{p}_{3}(1, \lambda, 0), \mathfrak{p}_{4}(1, \lambda, 0)\right)\left(\lambda_{0}\right)=0\right\} .
$$

There exists $i$ such that $\mathfrak{p}_{i}(1, \lambda, 0)$ is not identically zero, since otherwise $\mathfrak{v}$ would divide $\operatorname{gcd}\left(\mathfrak{p}_{1}, \ldots, \mathfrak{p}_{4}\right)$, which is a contradiction. Let us assume w.l.o.g. that $\mathfrak{p}_{4}(1, \lambda, 0)$ is not identically zero. We then introduce the finite set

$$
\mathcal{E}^{* *}=\left\{\left(1: \lambda_{0}: 0\right) \mid \mathfrak{p}_{4}\left(1, \lambda_{0}, 0\right)=0\right\} \backslash \mathcal{E}^{*},
$$

and we proceed to compute the multiplicity of each $\left(1: \lambda_{0}: 0\right) \in \mathcal{E}^{* *}$. For that, we observe that there exists $j \neq 4$ such that $\mathfrak{p}_{j}\left(1, \lambda_{0}, 0\right) \neq 0$, and we apply the multiplicity formula using the dehomogenization of $\mathcal{P}(\mathfrak{s}, \mathfrak{t}, \mathfrak{v})$ w.r.t. the $j$-component.

To analyze the open subset $\mathcal{E} \backslash \mathcal{E}^{* *}$, we work with the new affine parametrizations of $\mathcal{S}_{4}$, namely the parametrization obtained by taking $\mathfrak{s}=1$ and dehomogenizing $\mathcal{P}(\mathfrak{s}, \mathfrak{t}, \mathfrak{v})$ w.r.t. to the fourth component. We denote it by $\mathcal{P}_{4}^{\mathfrak{s}}(s, t)$. Similarly, if necessary, we introduce $\mathcal{P}_{i}^{\mathfrak{s}}(s, t)$ with $i=1,2,3$. Now, one has to proceed as in Section 4 , with the rational curve $\mathcal{Q}(\lambda)=(\lambda, 0)$.

\section{Algorithm}

In the following, we summarize all the previous ideas to derive an algorithm. For this purpose, let $\mathcal{S} \subset \mathbb{P}^{3}(\mathbb{K})$ be a rational projective surface, different to a plane, and $\mathcal{P}(\mathfrak{s}, \mathfrak{t}, \mathfrak{v})$ a parametrization of $\mathcal{S}$ expressed as

$$
\mathcal{P}(\mathfrak{s}, \mathfrak{t}, \mathfrak{v})=\left(\mathfrak{p}_{1}(\mathfrak{s}, \mathfrak{t}, \mathfrak{v}): \mathfrak{p}_{2}(\mathfrak{s}, \mathfrak{t}, \mathfrak{v}): \mathfrak{p}_{3}(\mathfrak{s}, \mathfrak{t}, \mathfrak{v}): \mathfrak{p}_{4}(\mathfrak{s}, \mathfrak{t}, \mathfrak{v})\right)
$$

where $\mathfrak{p}_{i} \in \mathbb{K}[\mathfrak{s}, \mathfrak{t}, \mathfrak{v}]$ are homogeneous polynomials of the same degree, and $\operatorname{gcd}\left(\mathfrak{p}_{1}, \mathfrak{p}_{2}, \mathfrak{p}_{3}, \mathfrak{p}_{4}\right)=1$. Let $\mathfrak{B}$ the zero set in $\mathbb{P}^{2}(\mathbb{K})$ of $\left\{\mathfrak{p}_{1}, \ldots, \mathfrak{p}_{4}\right\}$. Then, the algorithm decomposes $\mathbb{P}^{2}(\mathbb{K}) \backslash \mathfrak{B}$ as

$$
\mathbb{P}^{2}(\mathbb{K}) \backslash \mathfrak{B}=\bigcup_{k=1}^{\ell} \mathfrak{S}_{k}
$$

such that, if $\left(\mathfrak{s}_{0}: \mathfrak{t}_{0}: \mathfrak{v}_{0}\right) \in \mathfrak{S}_{k}$, then $\mathcal{P}\left(\mathfrak{s}_{0}, \mathfrak{t}_{0}, \mathfrak{v}_{0}\right)$ is a point of $\mathcal{S}$ of multiplicity $k$.

As already remarked in Section 2, we assume that none of the polynomials $\mathfrak{p}_{i}$ is zero or, more generally, that there do not exist $\mathfrak{p}_{i}, \mathfrak{p}_{j}$ and $\lambda \in \mathbb{K}$ such that $\mathfrak{p}_{i}=\lambda \mathfrak{p}_{j}$. Note that this excluded situation corresponds to a plane, and hence $\mathfrak{S}_{1}=\mathbb{P}^{2}(\mathbb{K})$.

In addition, we use the notation introduced in Section 2, namely, the affine surfaces $\mathcal{S}_{i}$, as well as and the affine rational parametrizations $\mathcal{P}_{i}(s, t), \mathcal{P}_{i}^{\mathfrak{t}}(s, t), \mathcal{P}_{i}^{\mathfrak{s}}(s, t)$ (see the subsection on singularities at infinity in Section 5). In this situation, the algorithm is as follows. 


\section{Algorithm}

Input: A projective rational surface $\mathcal{S} \subset \mathbb{P}^{3}(\mathbb{K})$, different to a plane, parametrized by

$$
\mathcal{P}(\mathfrak{s}, \mathfrak{t}, \mathfrak{v})=\left(\mathfrak{p}_{1}(\mathfrak{s}, \mathfrak{t}, \mathfrak{v}): \mathfrak{p}_{2}(\mathfrak{s}, \mathfrak{t}, \mathfrak{v}): \mathfrak{p}_{3}(\mathfrak{s}, \mathfrak{t}, \mathfrak{v}): \mathfrak{p}_{4}(\mathfrak{s}, \mathfrak{t}, \mathfrak{v})\right)
$$

where $\mathfrak{p}_{i} \in \mathbb{K}[\mathfrak{s}, \mathfrak{t}, \mathfrak{v}]$ are homogeneous polynomials of the same degree, and $\operatorname{gcd}\left(\mathfrak{p}_{1}, \mathfrak{p}_{2}\right.$, $\left.\mathfrak{p}_{3}, \mathfrak{p}_{4}\right)=1$.

Output: A decomposition of $\mathbb{P}^{2}(\mathbb{K}) \backslash \mathfrak{B}\left(\mathfrak{B}\right.$ is the zero set in $\mathbb{P}^{2}(\mathbb{K})$ of $\left.\left\{\mathfrak{p}_{1}, \ldots, \mathfrak{p}_{4}\right\}\right)$ as

$$
\mathbb{P}^{2}(\mathbb{K}) \backslash \mathfrak{B}=\bigcup_{k=1}^{\ell} \mathfrak{S}_{k}
$$

such that, if $\left(\mathfrak{s}_{0}: \mathfrak{t}_{0}: \mathfrak{v}_{0}\right) \in \mathfrak{S}_{k}$, then $\mathcal{P}\left(\mathfrak{s}_{0}, \mathfrak{t}_{0}, \mathfrak{v}_{0}\right)$ is a point of $\mathcal{S}$ of multiplicity $k$.

\section{[Preparatory Steps]}

[Step 0] If any of the projective curves defined by the non-constant numerators or denominators of the parametrization $\mathcal{P}_{4}$ passes through $(0: 1: 0)$ we perform a suitable affine linear change in the parameters $\{s, t\}$.

[Step 1] Apply Method 2 to compute $n_{2}:=\operatorname{deg}\left(\mathcal{P}_{4}\right)$ (see Section 3).

[Step 2] Apply Method 5 to determine an affine point, say $A_{0} \in \mathbb{K}^{3}$, out of the affine surface $\mathcal{S}_{4}$ (see Section [3); take $A_{0}$ with non-zero components such that, if the algorithm, in subsequent steps, requires a point in $\mathbb{K}^{3} \backslash \mathcal{S}_{i}$ with $i \neq 4$ no further computation would be needed (see Remark [3.3), and apply Method 3 to compute $n_{1}:=\operatorname{deg}\left(\Phi_{2,3}\left(A_{0}\right)\right)$ (see Section $\underline{3}$ ).

[Step 3] For $i=1, \ldots, 4$, let $\Delta_{i}$ be as in Lemma 2.2, and $\mathfrak{B}_{a}=\bigcap_{i=1}^{4} \Delta_{i}$.

[P-affine singularities (First level)]

[Step 4] Apply Method 8 to $\mathcal{S}_{4}, \mathcal{P}_{4}(s, t), \Gamma:=\mathbb{K}^{2}, A_{0}$ (see Step 2) and $n_{1}, n_{2}$ (see Steps 1 and 2). Let $\mathbb{K}^{2} \backslash \Delta_{4}=\bigcup_{k=1}^{\ell} \mathfrak{F}_{k}$ be the output of Method 8, then $\mathbf{j}\left(\mathfrak{F}_{k}\right) \subset \mathfrak{S}_{k}$ (see Section 4).

[P -affine singularities (Second, Third and Fourth Level)]

[Step 5] If $\Delta_{4} \backslash \mathfrak{B}_{a}=\emptyset$ (see Step 3) go to Step 8 else proceed as follows:

[Step 5.1] If $\Delta_{4} \backslash \Delta_{3}=\emptyset$ go to Step 6 .

[Step 5.2] Apply Method 8 taking: $\mathcal{P}_{3}(s, t)$ instead of $\mathcal{Q}(s, t), \mathcal{S}_{3}$ instead of $\mathcal{Z}$, and $\Gamma$ as $\Delta_{4} \backslash \Delta_{3}$. For those sets $\mathfrak{F}_{k}$ generated by this process, do $\mathbf{j}\left(\mathfrak{F}_{k}\right) \subset \mathfrak{S}_{k}$.

[Step 6] If $\left(\Delta_{4} \cap \Delta_{3}\right) \backslash \mathfrak{B}_{a}=\emptyset$ go to Step 8 else proceed as follows:

[Step 6.1] If $\left(\Delta_{4} \cap \Delta_{3}\right) \backslash \Delta_{2}=\emptyset$ go to Step 7 .

[Step 6.2] Apply Method 8 taking: $\mathcal{P}_{2}(s, t)$ instead of $\mathcal{Q}(s, t), \mathcal{S}_{2}$ instead of $\mathcal{Z}$, and $\Gamma$ as $\left(\Delta_{4} \cap \Delta_{3}\right) \backslash \Delta_{2}$. For those sets $\mathfrak{F}_{k}$ generated by this process, do $\mathbf{j}\left(\mathfrak{F}_{k}\right)$ $\subset \mathfrak{S}_{k}$.

[Step 7] If $\left(\Delta_{4} \cap \Delta_{3} \cap \Delta_{2}\right) \backslash \mathfrak{B}_{a}=\emptyset$ go to Step 8 else proceed as follows:

[Step 7.1] If $\left(\Delta_{4} \cap \Delta_{3} \cap \Delta_{2}\right) \backslash \Delta_{1}=\emptyset$ go to Step 8 .

[Step 7.2] Apply Method 8 taking: $\mathcal{P}_{1}(s, t)$ instead of $\mathcal{Q}(s, t), \mathcal{S}_{1}$ instead of $\mathcal{Z}$, and $\Gamma$ as $\left(\Delta_{4} \cap \Delta_{3} \cap \Delta_{2}\right) \backslash \Delta_{1}$. For those sets $\mathfrak{F}_{k}$ generated by this process, do $\mathbf{j}\left(\mathfrak{F}_{k}\right) \subset \mathfrak{S}_{k}$. 
[ $\mathcal{P}$ singularities at infinity]

[Step 8] If $(0: 1: 0) \notin \mathfrak{B}$ apply Method 6 to compute $\alpha:=\operatorname{mult}\left(\mathcal{P}_{4}^{\mathfrak{t}}(0,0), \mathcal{S}_{4}\right)$ and include $(0: 1: 0)$ in $\mathfrak{S}_{\alpha}$; we are assuming that $\mathfrak{p}_{4}(0,1,0) \neq 0$, otherwise take other $\mathfrak{p}_{i}$, say $\mathfrak{p}_{i_{0}}$, such that $\mathfrak{p}_{i_{0}}(0,1,0) \neq 0$, and work with $\mathfrak{p}_{i_{0}}$ instead of $\mathfrak{p}_{4}$.

[Step 9] Check whether $\mathfrak{p}_{4}(1, \lambda, 0)$ is not identically zero. If it does vanish, find $\mathfrak{p}_{i}$ not vanishing at $(1: \lambda: 0)$, say $\mathfrak{p}_{i_{0}}$, and in the following steps work with $\mathfrak{p}_{i_{0}}$ instead of $\mathfrak{p}_{4}$. Set $\mathcal{E}^{*}:=\left\{\left(1, \lambda_{0}\right) \mid \operatorname{gcd}\left(\mathfrak{p}_{1}(1, \lambda, 0), \mathfrak{p}_{2}(1, \lambda, 0), \mathfrak{p}_{3}(1, \lambda, 0), \mathfrak{p}_{4}(1, \lambda, 0)\right)\left(\lambda_{0}\right)=0\right\}$, and $\mathcal{E}^{* *}:=\left\{\left(1, \lambda_{0}\right) \mid \mathfrak{p}_{4}\left(1, \lambda_{0}, 0\right)=0\right\} \backslash \mathcal{E}^{*}$.

[Step 10] For each $\left(1: \lambda_{0}: 0\right)$ such that $\left(1, \lambda_{0}\right) \in \mathcal{E}^{* *}$, since $\left(1: \lambda_{0}: 0\right) \notin \mathfrak{B}$ find $\mathfrak{p}_{j}$ such that $\mathfrak{p}_{j}\left(1, \lambda_{0}, 0\right) \neq 0$, and compute $\alpha:=\operatorname{mult}\left(\mathcal{P}_{j}^{\mathfrak{s}}\left(\lambda_{0}, 0\right), \mathcal{S}_{j}\right)$. Include $\left(1: \lambda_{0}: 0\right)$ in $\mathfrak{S}_{\alpha}$.

[Step 11] Apply Method 8 taking: $\mathcal{P}_{4}^{\mathfrak{s}}(s, t)$ instead of $\mathcal{Q}(s, t), \mathcal{S}_{4}$ instead of $\mathcal{Z}$ and $\Gamma$ as $\left\{\left(1, \lambda_{0}\right) \mid \lambda_{0} \in \mathbb{K}\right\} \backslash \mathcal{E}^{* *}$. For those sets $\mathfrak{F}_{k}$ generated by this process, do $\mathbf{j}\left(\mathfrak{F}_{k}\right) \subset \mathfrak{S}_{k}$.

\section{EXAMPLES}

In this section we illustrate our algorithm by two examples. The first one is a 2 degree surface parametrization, while the second one is a bicubic. In the first example the (parameter) singular locus is real while, in the second, two complex lines and a triple real singularity appear. Computations in the examples were carried out with the mathematical software Maple. We have implemented some modules of the algorithm, but we do not have a self-contained implementation.

Example 6.1. We consider the parametrization

$$
\mathcal{P}(\mathfrak{s}, \mathfrak{t}, \mathfrak{v})=\left(\mathfrak{t}^{2}: \mathfrak{t}^{2}+\mathfrak{s}^{2}+\mathfrak{v}^{2}:(\mathfrak{s}+2 \mathfrak{t}) \mathfrak{v}:(\mathfrak{t}+\mathfrak{s}) \mathfrak{v}\right)
$$

of the complex projective surface $\mathcal{S}$. One can easily check that $\mathcal{S}$ is not a plane. In addition, the four affine parametrizations associated to the projective parametrization, as described in Section 2, are

$$
\begin{aligned}
& \mathcal{P}_{1}=\left(\frac{t^{2}+s^{2}+1}{t^{2}}, \frac{s+2 t}{t^{2}}, \frac{s+t}{t^{2}}\right), \quad \mathcal{P}_{3}=\left(\frac{t^{2}}{s+2 t}, \frac{t^{2}+s^{2}+1}{s+2 t}, \frac{t+s}{s+2 t}\right), \\
& \mathcal{P}_{2}=\left(\frac{t^{2}}{t^{2}+s^{2}+1}, \frac{s+2 t}{t^{2}+s^{2}+1}, \frac{s+t}{t^{2}+s^{2}+1}\right), \quad \mathcal{P}_{4}=\left(\frac{t^{2}}{s+t}, \frac{t^{2}+s^{2}+1}{s+t}, \frac{s+2 t}{s+t}\right) .
\end{aligned}
$$

We apply the algorithm. $\mathcal{P}_{4}$ satisfies the hypotheses in Step 0. In Step 1, one gets $n_{2}:=1$, and in Step 2 we get $A_{0}:=(1,1,1)$ and $n_{1}:=4$. In Step 3, we get that

- $\Delta_{1}$ is the line $t=0$,

- $\Delta_{2}$ is the complex circle $t^{2}+s^{2}=-1$,
- $\Delta_{3}$ is the line $s+2 t=0$,

- $\Delta_{4}$ is the line $s+t=0$,

that are the curves defined by the denominators of $\mathcal{P}_{i}(s, t)$. Note that $\mathfrak{B}_{a}=$ $\mathfrak{B}=\emptyset$. In Step 4, we apply Method 8 with the following input: $\mathcal{S}_{4}, A_{0}=$ $(1,1,1), \mathcal{P}_{4}(s, t), \Gamma:=\mathbb{C}^{2}, n_{2}=1, n_{1}=4$.

\section{[Execution of Method 8, for Step 4, starts]}

Since $\operatorname{dim}(\Theta)=2$, we skip Step 0 of Method 8. In Step 1 of Method 8, we apply Method 7 to compute $\Omega$ (in the next, we refer to steps in Method 7):

○ In Step 2, we get that $\Omega_{0}=\emptyset$. In Step 3, we compute $\Omega_{1}$. In Step 3.1, we get that $\mathcal{N}_{\infty}$ is union of the lines $t^{*}+s^{*}=0$ and $s^{*}=0$. In Step 3.2, we 
get $M_{1}=-s^{*}-t^{*}+\mathrm{X}_{1}\left(s^{*}+t^{*}\right), M_{2}=\mathrm{X}_{2}\left(s^{*}+t^{*}\right)$. So, in Step 3.3, we conclude that

$$
\Omega_{1}=\left\{\left(s^{*}, t^{*}\right) \in \mathbb{C}^{2} \mid t^{*}+s^{*}=0\right\} \cup\left\{\left(s^{*}, t^{*}\right) \in \mathbb{C}^{2} \mid s^{*}=0\right\} .
$$

○ For the computation of $\Omega_{2}$, in Step 4.1 , we get $W=\mathrm{X}_{2}^{2}\left(s^{*}+t^{*}\right)^{4}$. Therefore,

$$
\Omega_{2}=\left\{\left(s^{*}, t^{*}\right) \in \mathbb{C}^{2} \mid t^{*}+s^{*}=0\right\} .
$$

○ For computing $\Omega_{3}$ and $\Omega_{4}$, in Step 5.1, we get that $R$ has 6 non-zero coefficients w.r.t. $\left\{\mathrm{X}_{1}, \mathrm{X}_{2}\right\}$. Moreover, $a=\left(s^{*}+t^{*}\right)^{2}\left(s-s^{*}\right)$ (see Step 5.2) and $N=\left(s^{*}+t^{*}\right)^{2}$ (see Step 5.3). Since $\operatorname{Card}(J)=6$ we go through Step 5.5.

Then (see Step 5.5.1), $a_{i_{1}, j_{1}}=s\left(s^{* 2}+t^{* 2}\right)-s^{*}, L=s^{* 2}+t^{* 2}$, and

$$
\begin{aligned}
\bar{S}= & 2 t^{* 3} s^{* 4}+t^{*} s^{* 6}+2 t^{* 3} W_{1} s^{* 4}+2 t^{* 5} W_{1} s^{* 2}+3 s^{* 6} t^{* 3} W_{1}+s^{* 8} W_{1} t^{*} \\
& +3 s^{* 4} t^{* 5} W_{1}-2 s^{* 5} W_{2} t^{*}+s^{* 6} W_{4}+s^{* 8} W_{2}-s^{* 8} W_{4}-2 s^{* 7} W_{1}+t^{* 5} s^{* 2} \\
& -2 s^{* 3} W_{2} t^{* 3}+2 s^{* 2} W_{2} t^{* 4}+2 s^{* 4} W_{2} t^{* 2}-W_{4} s^{* 2} t^{* 4}+2 W_{4} s^{* 3} t^{* 3}+W_{1} s^{* 4} t^{*} \\
& +5 s^{* 4} W_{2} t^{* 4}+4 s^{* 6} W_{2} t^{* 2}-s^{* 6} W_{3} t^{*}-2 s^{* 4} W_{3} t^{* 3}-3 s^{* 6} W_{4} t^{* 2}-3 s^{* 4} W_{4} t^{* 4} \\
& +2 s^{* 5} W_{4} t^{*}-4 s^{* 5} W_{1} t^{* 2}+t^{* 7} W_{1} s^{* 2}+2 t^{* 6} s^{* 2} W_{2}-t^{* 5} W_{3} s^{* 2}-t^{* 6} W_{4} s^{* 2} \\
& -2 t^{* 4} W_{1} s^{* 3}-2 W_{1} s^{* 3} t^{* 2}+W_{1} s^{* 2} t^{* 3} .
\end{aligned}
$$

In Step 5.5.2 and Step 5.5.3, we get

$$
\begin{gathered}
\Omega_{3}=\left\{\left(s^{*}, t^{*}\right) \in \mathbb{C}^{2} \mid t^{*}=0\right\}, \\
\Omega_{4}=\left\{\left(s^{*}, t^{*}\right) \in \mathbb{C}^{2} \mid s^{*^{2}}+t^{* 2}=0\right\} \cup\left\{\left(s^{*}, t^{*}\right) \in \mathbb{C}^{2} \mid t^{*}+s^{*}=0\right\} .
\end{gathered}
$$

- In Step 6 of Method 7, we get

$$
\begin{aligned}
\Omega= & \bigcup_{i=0}^{4} \Omega_{i}=\left\{\left(s^{*}, t^{*}\right) \in \mathbb{C}^{2} \mid t^{*}+s^{*}=0\right\} \cup\left\{\left(s^{*}, t^{*}\right) \in \mathbb{C}^{2} \mid s^{*}=0\right\} \\
& \cup\left\{\left(s^{*}, t^{*}\right) \in \mathbb{C}^{2} \mid t^{*}=0\right\} \cup\left\{\left(s^{*}, t^{*}\right) \in \mathbb{C}^{2} \mid s^{* 2}+t^{* 2}=0\right\} .
\end{aligned}
$$

At this point, we have computed the main set of simple points, that is, we know that parameter values in $\mathbb{C}^{2} \backslash \Omega$ are simple. We proceed to study the parameter values in $\Omega$. Step 3 (Method 8) is not needed, and in Step 4 (Method 8) we get that all components are rational; indeed lines. So we go through the genus zero case. Then, for each of the lines we execute Step 4 (Method 8) that implies the application of Method 6 (below, we refer to steps in Method 6):

- Let $\mathcal{C}$ be the line $t^{*}=0$. We consider the normal proper parametrization

$$
\mathcal{Q}(\lambda)=(\lambda, 0), \text { and } \mathcal{H}(\lambda)=\left(0, \frac{1+\lambda^{2}}{\lambda}, 1\right)
$$

Now, we apply Method 6 to the generic point $\mathcal{H}(\lambda) . \quad A_{0}=(1,1,1)$ was already computed. Moreover, since $\Omega_{0}=\emptyset$, then $\mathfrak{g}^{\mathcal{H}(\lambda)}=1$ for $\lambda \neq 0$. So, we perform Step 4 (Method 6) dealing generically with $\mathcal{H}(\lambda)$. We already know that $n_{1}=4$ and $n_{2}=1$. So it only remains to compute $m_{1}$ (see Step 4.1, Method 6), that is, to apply Method 3 to compute $\operatorname{deg}\left(\Phi_{2,3}(\mathcal{H}(\lambda))\right)$. We observe that Step 0 (Method 3) holds for all $\lambda \neq 0$. We get PrimPart $\operatorname{PX}_{\left.1, X_{2}\right\}}\left(R\left(s, X_{1}, X_{2}\right)\right)=X_{2}^{2} \lambda^{2} s-X_{2}^{2} \lambda s^{2}-X_{2}^{2} \lambda+X_{2}^{2} s+$ $X_{2} \lambda^{2}+X_{1} \lambda+X_{2}-\lambda$ (see Steps 3 and 4 in Method 3 ), and hence $m_{1}=2$ 
for $\lambda \neq 0$; note that for this particular $\mathcal{H}(\lambda)$ all steps specialize properly when $\lambda \neq 0$. Thus, Method 6 outputs

$$
\operatorname{mult}((\lambda: 0: 1))=\operatorname{mult}\left(\mathcal{H}(\lambda), \mathcal{S}_{4}\right)=\frac{n_{1}-m_{1}}{n_{2}}=2 \text {, with } \lambda \neq 0 \text {. }
$$

So, we have computed the multiplicity for affine parameter values in $\mathcal{C} \backslash$ $\{(0,0)\}$, and, with the exception of one point that we have not analyzed yet, this line is of double points.

- Let $\mathcal{C}$ be the line $s^{*}=0$. We consider the normal proper parametrization

$$
\mathcal{Q}(\lambda)=(0, \lambda), \text { and } \mathcal{H}(\lambda)=\left(\lambda, \frac{1+\lambda^{2}}{\lambda}, 2\right) .
$$

We reason as above. But, when applying Step 0 of Method 3, one realizes that a linear change in $\{s, t\}$ is required. We change $(s, t)$ by $(s+t, t-s)$. This implies that $\mathcal{Q}(\lambda)$ and $\mathcal{H}(\lambda)$ change to

$$
\mathcal{Q}(\lambda)=(\lambda,-\lambda), \text { and } \mathcal{H}(\lambda)=\left(-2 \lambda, \frac{4 \lambda^{2}+1}{-2 \lambda}, 2\right) .
$$

In this situation, one gets that $m_{1}=3$ for $\lambda \neq 0$. Thus, Method 6 outputs

$$
\operatorname{mult}((0: \lambda: 1))=\frac{n_{1}-m_{1}}{n_{2}}=1, \text { with } \lambda \neq 0 .
$$

So, we have computed the multiplicity for affine parameter values in $\mathcal{C} \backslash$ $\{(0,0)\}$, and, with the exception of one point that we have not analyzed yet, this line is of simple points.

- The next curve is precisely $\Delta_{4}$. So, we postpone its analysis to further levels.

- Let $\mathcal{C}$ be the lines $s^{*} \pm \imath t^{*}=0$; we treat both curves simultaneously. We consider the normal proper parametrization

$$
\mathcal{Q}(\lambda)=( \pm \imath \lambda, \lambda) \text {, and } \mathcal{H}(\lambda)=\left(\frac{\lambda^{2}}{\lambda \pm \imath \lambda}, \frac{1}{\lambda \pm \imath \lambda}, \frac{ \pm \imath \lambda+2 \lambda}{\lambda \pm \imath \lambda}\right)
$$

Reasoning as above, we get that $m_{1}=3$. Therefore, $\operatorname{mult}(( \pm \imath \lambda: \lambda: 1))=$ 1 , for $\lambda \neq 0$. So, we have computed the multiplicity for affine parameter values in $\mathcal{C} \backslash\{(0,0)\}$, and, with the exception of one point that we have not analyzed yet, these two lines are of simple points.

[Execution of Method 8 ends, and hence Step 4 of the algorithm]

At this point, we have analyzed $\mathbb{C}^{2} \backslash \Delta_{4}$, that is, all affine points but those where the denominator of $\mathcal{P}_{4}(s, t)$ vanishes. In order to study these points, we continue with Step 5 of the algorithm. $\Delta_{4} \cap \Delta_{3}=\{(0,0)\}$. Since $\Delta_{4} \backslash \mathcal{B}_{a} \neq \emptyset$ and $\Delta_{4} \backslash \Delta_{3} \neq \emptyset$ we go to Step 5.2 of the algorithm. Thus, we apply Method 8 with input: $\mathcal{P}_{3}(s, t), \mathcal{S}_{3}, \Gamma:=\Delta_{4} \backslash \Delta_{3}, A_{0}=(1,1,1), n_{1}=4, n_{2}=1$.

\section{[Execution of Method 8, for Step 5.2, starts]}

Since $\operatorname{dim}(\Theta)=1$, we apply Step 4 of Method 8 to the line $\Delta_{4}$ that implies the application of Method 6. For this purpose, we consider the parametrization $\mathcal{Q}(\lambda)=(-\lambda, \lambda)$ and $\mathcal{H}(\lambda)=\mathcal{P}_{3}(\mathcal{Q}(\lambda))$. Now, we apply Method 6 to the generic point $\mathcal{H}(\lambda) . A_{0}=(1,1,1)$ was already computed. Moreover, since $\Omega_{0}=\emptyset$, then $\mathfrak{g}^{\mathcal{H}(\lambda)}=1$ for $\lambda \neq 0$. So, we perform Step 4 (Method 6) dealing generically with $\mathcal{H}(\lambda)$. We already know that $n_{1}=4$ and $n_{2}=1$. So it only remains to compute $m_{1}$ 
(see Step 4.1, Method 6), that is, to apply Method 3 to compute $\operatorname{deg}\left(\Phi_{2,3}(\mathcal{H}(\lambda))\right)$. We observe that for this particular $\mathcal{H}(\lambda)$ all steps specialize properly when $\lambda \neq 0$. Thus, Method 6 outputs

$$
\operatorname{mult}((-\lambda: \lambda: 1))=\operatorname{mult}\left(\mathcal{H}(\lambda), \mathcal{S}_{2}\right)=\frac{n_{1}-m_{1}}{n_{2}}=1, \text { with } \lambda \neq 0 .
$$

So, we have computed the multiplicity for affine parameter values in $\Delta_{4} \backslash \Delta_{3}$, and, with the exception of one point that we still have not analyzed, the curve $\Delta_{4}$ is of simple points.

[Execution of Method 8 ends, and hence Step 5 of the algorithm]

It only remains, in terms of affine values, to analyze the origin. In Step 6 of the main algorithm, since $\left(\Delta_{4} \cap \Delta_{3}\right) \backslash \Delta_{2}=\{(0,0)\}$, we go to Step 6.2, and we compute the multiplicity of $\mathcal{P}_{2}(0,0)=(0,0,0)$ using $\mathcal{P}_{2}$. We get

$$
\operatorname{mult}((0: 0: 1))=\operatorname{mult}\left((0,0,0), \mathcal{S}_{2}\right)=3 \text {. }
$$

Since $\Delta_{4} \cap \Delta_{3} \cap \Delta_{2}=\emptyset$, we skip Step 7, and we pass to Step 8. Therefore, we have finished the analysis of the affine points and we study the points at infinity. For this purpose, in Step 8, we first observe that $\mathfrak{B}=\emptyset$. Moreover, since $\mathfrak{p}_{4}(0,1,0)=0$ but $\mathfrak{p}_{1}(0,1,0) \neq 0$ we compute $\operatorname{mult}\left(\mathcal{P}_{1}^{\mathfrak{t}}(0,0), \mathcal{S}_{1}\right)$ by applying Method 6 . One gets that $m_{1}:=\operatorname{deg}\left(\Phi_{2,3}\left(\mathcal{P}_{1}^{\mathfrak{t}}(0,0)\right)\right)=2$. So,

$$
\operatorname{mult}((0: 1: 0))=\operatorname{mult}\left(\mathcal{P}_{1}^{\mathfrak{t}}(0,0), \mathcal{S}_{1}\right)=\frac{n_{1}-m_{1}}{n_{2}}=2 .
$$

In Step 9, we observe that $\mathfrak{p}_{4}(1, \lambda, 0)=0$ but $\mathfrak{p}_{1}(1, \lambda, 0)=\lambda^{2}$. So, in the following, we work with $\mathfrak{p}_{1}$ instead of $\mathfrak{p}_{4}$. Moreover, we get that $\mathcal{E}^{*}=\emptyset$, and $\mathcal{E}^{* *}=\{(1,0)\}$. In Step 10, we need to analyze $(1: 0: 0)$. We do it using $\mathcal{P}_{2}^{\mathfrak{s}}(s, t)$ to get

$$
\operatorname{mult}((1: 0: 0))=\operatorname{mult}\left((0,0,0), \mathcal{S}_{2}\right)=3 \text {. }
$$

In Step 11, we apply Method 8 with input: $\mathcal{S}_{1}, \mathcal{P}_{1}^{\mathfrak{s}}(s, t), A_{0}=(1,1,1), n_{1}=4, n_{2}=$ 1 , and $\Gamma=\{(1, \lambda) \mid \lambda \neq 0\}$. We get

$$
\operatorname{mult}((1: \lambda: 0))=\operatorname{mult}\left(\left(\frac{1+\lambda^{2}}{\lambda^{2}}, 0,0\right), \mathcal{S}_{1}\right)=2, \text { for } \lambda \notin\{0,1,-1\} \text {. }
$$

So it only remains to analyze $(1: 1: 0),(1:-1: 0)$. We apply Method 6 with $\mathcal{P}_{1}$ to get

$$
\operatorname{mult}((1: 1: 0))=1, \quad \operatorname{mult}((1:-1: 0))=1 .
$$

Summarizing $\mathbb{P}^{2}(\mathbb{C})$ is decomposed as $\mathbb{P}^{2}(\mathbb{C})=\cup_{k=1}^{3} \mathfrak{S}_{k}$, where

(1) $\mathfrak{S}_{1}=\{(\mathfrak{s}: \mathfrak{t}: 1) \mid \mathfrak{t} \neq 0\}$,

(2) $\mathfrak{S}_{2}=\{(\mathfrak{s}: \mathfrak{t}: 0) \mid \mathfrak{t} \neq 0\} \cup\{(\mathfrak{s}: 0: 1) \mid \mathfrak{s} \neq 0\}$,

(3) $\mathfrak{S}_{3}=\{(1: 0: 0),(0: 0: 1)\}$.

Example 6.2. We consider the parametrization

$$
\begin{aligned}
\mathcal{P}(\mathfrak{s}, \mathfrak{t}, \mathfrak{v})=\left(198 \mathfrak{s}^{3}+196 \mathfrak{t}^{3}-\right. & 372 \mathfrak{v}^{3}:-89 \mathfrak{s}^{3}-89 \mathfrak{t}^{3}+135 \mathfrak{v}^{3} \\
: & \left.189 \mathfrak{t}^{3}+3 \mathfrak{t}^{2} \mathfrak{s}+3 \mathfrak{s t}^{2}+189 \mathfrak{s}^{3}-372 \mathfrak{v}^{3}: \mathfrak{s}^{3}+\mathfrak{t}^{3}\right)
\end{aligned}
$$


of the complex projective surface $\mathcal{S}$. One can easily check that $\mathcal{S}$ is not a plane. In addition, the four affine parametrization associated to the projective parametrization, as described in Section 2, are

$$
\begin{gathered}
\mathcal{P}_{1}=\left(\frac{-89 s^{3}-89 t^{3}+135}{198 s^{3}+196 t^{3}-372}, \frac{189 t^{3}+3 t^{2} s+3 t s^{2}+189 s^{3}-372}{198 s^{3}+196 t^{3}-372},\right. \\
\mathcal{P}_{2}=\left(\frac{198 s^{3}+196 t^{3}-372}{-89 s^{3}-89 t^{3}+135}, \frac{189 t^{3}+3 t^{2} s+3 t s^{2}+189 s^{3}-372}{-89 s^{3}-89 t^{3}+135},\right. \\
\mathcal{P}_{3}=\left(\frac{196 t^{3}-372}{189 t^{3}+3 t^{2} s+3 t s^{2}+189 s^{3}-372}, \frac{s^{3}+t^{3}}{189 t^{3}+3 t^{2} s+3 t s^{2}+89 t^{3}+135}\right) \\
\mathcal{P}_{4}=\left(\frac{198 s^{3}+196 t^{3}-372}{s^{3}+t^{3}}, \frac{-89 s^{3}-89 t^{3}+135}{s^{3}+t^{3}},\right. \\
\left.\frac{189 t^{3}+3 t^{2} s+3 t s^{2}+189 s^{3}-372}{s^{3}+t^{3}}\right) .
\end{gathered}
$$

We apply the algorithm to $\mathcal{P}(\mathfrak{s}, \mathfrak{t}, \mathfrak{v}) . \quad \mathcal{P}_{4}$ satisfies the hypotheses in Step 0 . In Step 1 , one gets $n_{2}:=3$ (observe that this means that the parametrization is not proper), and in Step 2 we get $A_{0}:=(1,1,1)$ and $n_{1}:=9$. In Step 3, we get that

- $\Delta_{1}$ is the cubic $198 s^{3}+196 t^{3}-372=0$,

- $\Delta_{2}$ is the cubic $-89 s^{3}-89 t^{3}+135=0$,

- $\Delta_{3}$ is the cubic $189 t^{3}+3 t^{2} s+3 t s^{2}+189 s^{3}-372=0$, and

- $\Delta_{4}$ is the cubic $s^{3}+t^{3}=0$

are the curves defined by the denominators of $\mathcal{P}_{i}(s, t)$. Note that $\mathfrak{B}_{a}=\mathfrak{B}=\emptyset$. In Step 4, we apply Method 8 with the following input: $\mathcal{S}_{4}, \mathcal{P}_{4}(s, t), A_{0}=(1,1,1), n_{1}=$ $9, n_{2}=3, \Gamma=\mathbb{C}^{2}$.

\section{[Execution of Method 8, for Step 4, starts]}

Since $\operatorname{dim}(\Theta)=2$, we skip Step 0 of Method 8. In Step 1 of Method 8, we apply Method 7 to compute $\Omega$ (below, we refer to steps in Method 7):

○In Step 2, we get that $\Omega_{0}=\emptyset$. In Step 3 , we compute $\Omega_{1}$. We get that

$$
\Omega_{1}=\left\{\left(s^{*}, t^{*}\right) \in \mathbb{C}^{2} \mid\left(s^{* 3}-186\right)\left(-s^{*} t^{* 2}-s^{* 2} t^{*}+124\right)=0\right\} .
$$

○ Step 4 outputs $\Omega_{2}=\left\{\left(s^{*}, t^{*}\right) \in \mathbb{C}^{2} \mid t^{* 3}+s^{* 3}=0\right\}$.

- For computing $\Omega_{3}$ and $\Omega_{4}$, in Step 5.1, we get that $R$ has 10 non-zero coefficients w.r.t. $\left\{\mathrm{X}_{1}, \mathrm{X}_{2}\right\}$. Since $\operatorname{Card}(J)=10$ we go through Step 5.5, and in Step 5.5.2 and Step 5.5.3, we get

$$
\begin{gathered}
\Omega_{3}=\left\{\left(s^{*}, t^{*}\right) \in \mathbb{C}^{2} \mid\left(t^{* 2}+s^{*} t^{*}+s^{* 2}\right)\left(s^{*}+t^{*}\right)=0\right\}, \\
\Omega_{4}=\left\{\left(s^{*}, t^{*}\right) \in \mathbb{C}^{2} \mid t^{* 3}+s^{* 3}=0\right\} \cup\left\{\left(s^{*}, t^{*}\right) \in \mathbb{C}^{2} \mid s^{*}+t^{*}=0\right\} .
\end{gathered}
$$


○ In Step 6 of Method 7, we get

$$
\begin{aligned}
\Omega=\bigcup_{i=0}^{4} \Omega_{i}= & \left\{\left(s^{*}, t^{*}\right) \in \mathbb{C}^{2} \mid\left(s^{* 3}-186\right)\left(-s^{*} t^{* 2}-s^{* 2} t^{*}+124\right)=0\right\} \\
& \cup\left\{\left(s^{*}, t^{*}\right) \in \mathbb{C}^{2} \mid t^{* 3}+s^{* 3}=0\right\} \\
& \cup\left\{\left(s^{*}, t^{*}\right) \in \mathbb{C}^{2} \mid\left(t^{* 2}+s^{*} t^{*}+s^{* 2}\right)\left(s^{*}+t^{*}\right)=0\right\} \\
& \cup\left\{\left(s^{*}, t^{*}\right) \in \mathbb{C}^{2} \mid s^{*}+t^{*}=0\right\} .
\end{aligned}
$$

Therefore in Step 2 of Method 8, we get

$$
\begin{aligned}
\Omega= & \left\{\left(s^{*}, t^{*}\right) \in \mathbb{C}^{2} \mid s^{*}+t^{*}=0\right\} \cup\left\{\left(s^{*}, t^{*}\right) \in \mathbb{C}^{2} \mid s^{*} \pm \frac{1}{2}(\sqrt{3} \imath \mp 1) t^{*}=0\right\} \\
& \cup\left\{\left(s^{*}, t^{*}\right) \in \mathbb{C}^{2} \mid s^{*}-\frac{1}{2}(-1 \pm \sqrt{3} \imath) t^{*}=0\right\} \\
& \cup\left\{\left(s^{*}, t^{*}\right) \in \mathbb{C}^{2} \mid-s^{*} t^{* 2}-s^{* 2} t^{*}+124=0\right\} \\
& \cup\left\{\left(s^{*}, t^{*}\right) \in \mathbb{C}^{2} \mid s^{*}-186^{1 / 3}=0\right\} \\
& \cup\left\{\left(s^{*}, t^{*}\right) \in \mathbb{C}^{2} \mid s^{*}-\frac{186^{1 / 3}}{2}(-1 \pm \imath \sqrt{3})=0\right\} .
\end{aligned}
$$

At this point, we have computed the main set of simple points, that is, we know that parameter values in $\mathbb{C}^{2} \backslash \Omega$ are simple. We proceed to study the parameter values in $\Omega$. Step 3 (Method 8) is not needed, and in Step 4 (Method 8) we get that all components are rational (in fact, lines) except the cubic defined by the polynomial $-s^{*} t^{* 2}-s^{* 2} t^{*}+124$. So, in Step 4 (Method 8), we go through the genus zero case for each of the lines, and for the cubic, we work over the field of rational functions. In any case, this implies the application of Method 6 (in the following, we refer to steps in Method 6).

- The first two sets are included in $\Delta_{4}$. So, we postpone its analysis to further levels.

- Let $\mathcal{C}$ be the line $s^{*}-\frac{1}{2}(-1+\sqrt{3} \imath) t^{*}=0$; similarly for $s^{*}-\frac{1}{2}(-1-$ $\sqrt{3} \imath) t^{*}=0$. We consider the normal proper parametrization $\mathcal{Q}(\lambda)=$ $\left(\frac{1}{2}(-1+\sqrt{3} \imath) \lambda, \lambda\right)$, and

$$
\mathcal{H}(\lambda)=\left(\frac{197 \lambda^{3}-186}{\lambda^{3}}, \frac{-\left(178 \lambda^{3}-135\right)}{2 \lambda^{3}}, \frac{3\left(125 \lambda^{3}-124\right)}{2 \lambda^{3}}\right) .
$$

Now, we apply Method 6 to the generic point $\mathcal{H}(\lambda)$. This implies the application of Method 3 to compute $m_{1}:=\operatorname{deg}\left(\Phi_{2,3}(\mathcal{H}(\lambda))\right)$. We get $m_{1}=3$ for $\lambda \neq 0$; for the particular case where $\lambda$ is a root of $\lambda^{3}=186$ or of $3 \lambda^{3}+372=0$, the conditions in Step 0 (Method 3$)$ do not hold, and a linear transformation in $\{s, t\}$ is required getting also $m_{1}=3$. Summarizing, Method 6 outputs, for $\lambda \neq 0$,

$\operatorname{mult}(((-1 / 2 \pm \imath \sqrt{3} / 2) \lambda: \lambda: 1))=\operatorname{mult}\left(\mathcal{H}(\lambda), \mathcal{S}_{4}\right)=\frac{n_{1}-m_{1}}{n_{2}}=2$.

So, we have computed the multiplicity for affine parameter values in $\mathcal{C} \backslash$ $\{(0,0)\}$, and, with the exception of one point that we still have not analyzed, the line is of double points. 
- Let $\mathcal{C}$ be the line $s^{*}=\sqrt[3]{186}$. We consider the normal proper parametrization $\mathcal{Q}(\lambda)=(\sqrt[3]{186}, \lambda)$ and $\mathcal{H}(\lambda)=\mathcal{P}_{4}(\mathcal{Q}(\lambda)) ; \mathcal{H}(\lambda)$ is defined for $\lambda \neq$ $-\sqrt[3]{186}$. Now, we apply Method 6 to the generic point $\mathcal{H}(\lambda)$. This implies the application of Method 3 to compute $m_{1}:=\operatorname{deg}\left(\Phi_{2,3}(\mathcal{H}(\lambda))\right)$. Conditions in Step 0 (Method 3) do not hold, and a linear transformation in $\{s, t\}$ is required. We change $(s, t)$ by $(t, s)$. In this situation, the conditions in Step 0 (Method 3) hold if $\lambda$ is not a root of $372-3 \sqrt[3]{186}{ }^{2} \lambda-3 \sqrt[3]{186} \lambda^{2}=0$; these parameter values, i.e., for the roots of the previous polynomial, are treated directly using Method 6. Summarizing, we get that, for $\lambda \neq-\sqrt[3]{186}$, $m_{1}=6$, and hence Method 6 outputs

$$
\operatorname{mult}((\sqrt[3]{186}: \lambda: 1))=1, \text { with } \lambda \neq-\sqrt[3]{186}
$$

So, we have computed the multiplicity for affine parameter values in $\mathcal{C} \backslash$ $\{(\sqrt[3]{186},-\sqrt[3]{186})\}$, and, with the exception of one point that we still have not analyzed, the line is of simple points.

- Let $\mathcal{C}$ be the line $s^{*}=\frac{\sqrt[3]{186}}{2}(-1+\imath \sqrt{3})$; similarly for $s^{*}=\frac{\sqrt[3]{186}}{2}(-1-\imath \sqrt{3})$. We consider the normal proper parametrization

$$
\mathcal{Q}(\lambda)=\left(\frac{\sqrt[3]{186}}{2}(-1 \pm \sqrt{3}), \lambda\right) \text {, and } \mathcal{H}(\lambda)=\mathcal{P}_{4}(\mathcal{Q}(\lambda))
$$

$\mathcal{H}(\lambda)$ is defined for $\lambda \neq-\sqrt[3]{186}$. Now, we apply Method 6 to the generic point $\mathcal{H}(\lambda)$. This implies the application of Method 3 to compute $m_{1}:=$ $\operatorname{deg}\left(\Phi_{2,3}(\mathcal{H}(\lambda))\right)$. Conditions in Step 0 (Method 3) do not hold, and a linear transformation in $\{s, t\}$ is required. We change $(s, t)$ by $(t, s)$. In this situation, the conditions in Step 0 (Method 3) hold if $\lambda$ is not a root of $744+3 \imath \sqrt[3]{186}^{2} \sqrt{3} \lambda-3 \imath \sqrt[3]{186} \sqrt{3} \lambda^{2}+3 \sqrt[3]{186}^{2} \lambda+3 \sqrt[3]{186}^{2}=0$; these parameter values, i.e. for the roots of the previous polynomial, are treated directly using Method 6 . Summarizing we get that, for $\lambda \neq-\sqrt[3]{186}, m_{1}=6$, and hence Method 6 outputs

$$
\operatorname{mult}\left(\left(\frac{\sqrt[3]{186}}{2}(-1 \pm \imath \sqrt{3}): \lambda: 1\right)\right)=1, \text { with } \lambda \neq-\sqrt[3]{186}
$$

So, we have computed the multiplicity for affine parameter values in $\mathcal{C} \backslash$ $\left\{\left(\frac{\sqrt[3]{186}}{2}(-1 \pm \imath \sqrt{3}),-\sqrt[3]{186}\right)\right\}$, and, with the exception of two points that we still have not analyzed, these two lines are of simple points.

- Let $\mathcal{C}$ be the cubic $s^{*} t^{* 2}+s^{* 2} t^{*}=124$. In this case, since $\mathcal{C}$ is not rational, we work over the field of rational functions $\mathbb{C}(\mathcal{C})$. For this purpose, we first consider $\mathcal{Q}(\lambda, \beta)=(\lambda, \beta)$, and $\mathcal{H}(\lambda, \beta)=\mathcal{P}_{4}(\lambda, \beta)$, where $f(\lambda, \beta)=$ $-\lambda \beta^{2}-\lambda^{2} \beta+124$, and we apply Method 8 replacing $\mathcal{A}$ by $\mathcal{H}(\lambda, \beta)$, where $f(\lambda, \beta)=0$. Reasoning as in the previous cases, but using the arithmetic in the field $\mathbb{C}(\mathcal{C})$ (that is, we compute remainders with the polynomial $f(\lambda, \beta))$, we get that

$\operatorname{mult}((\lambda: \beta: 1))=\operatorname{mult}\left(\mathcal{H}(\lambda, \beta), \mathcal{S}_{4}\right)=1$, with $(\lambda, \beta)$ such that $f(\lambda, \beta)=0$.

So, also, points in the cubic are simple points. 


\section{[Execution of Method 8 ends, and hence Step 4 of the algorithm]}

At this point, we have analyzed $\mathbb{C}^{2} \backslash \Delta_{4}$; observe that the excluded points in the above analysis, namely $(0,0),(\sqrt[3]{186},-\sqrt[3]{186}),\left(\frac{\sqrt[3]{186}}{2}(-1 \pm \imath \sqrt{3}),-\sqrt[3]{186}\right)$, belong to $\Delta_{4}$. In order to analyze the points of the denominator curve $\Delta_{4}$, we continue with Step 5 of the algorithm. Since $\Delta_{4} \backslash \mathcal{B}_{a} \neq \emptyset$ and $\Delta_{4} \backslash \Delta_{3} \neq \emptyset$ we go to Step 5.2 of the algorithm. Thus, we apply Method 8 with input: $\mathcal{P}_{3}(s, t), \mathcal{S}_{3}, \Gamma:=\Delta_{4} \backslash \Delta_{3}, A_{0}=$ $(1,1,1), n_{1}=9, n_{2}=3$.

\section{[Execution of Method 8, for Step 5.2, starts]}

Since $\operatorname{dim}(\Theta)=1$, we apply Step 4 of Method 8 to $\Delta_{4}$ that implies the application of Method 6. Observe that $\Delta_{4}$ is the union of the lines $s+t=0, s+(1 / 2)(\sqrt{-3}-$ $1) t=0$ and $s-(1 / 2)(\sqrt{-3}+1) t=0$. For this purpose, and for each line, we consider the parametrization $\mathcal{Q}(\lambda)$ of the line and $\mathcal{H}(\lambda)=\mathcal{P}_{3}(\mathcal{Q}(\lambda))$. Then, we apply Method 6 to the generic point $\mathcal{H}(\lambda) . A_{0}=(1,1,1)$ was already computed. Moreover, since $\Omega_{0}=\emptyset$, then $\mathfrak{g}^{\mathcal{H}(\lambda)}=1$ for $\lambda \neq 0$. So, we perform Step 4 (Method 6 ) dealing generically with $\mathcal{H}(\lambda)$. We already know that $n_{1}=9$ and $n_{2}=3$. So it only remains to compute $m_{1}$ (see Step 4.1, Method 6), that is, to apply Method 3 to compute $\operatorname{deg}\left(\Phi_{2,3}(\mathcal{H}(\lambda))\right)$.

- Line $s+t=0$. Then, $\mathcal{Q}(\lambda)=(\lambda,-\lambda)$ and $\mathcal{H}(\lambda)=\mathcal{P}_{3}(\mathcal{Q}(\lambda))=\left(-(1 / 186) \lambda^{3}\right.$ $+1,-45 / 124,0)$. We observe that for this particular $\mathcal{H}(\lambda)$ all steps specialize properly with the exception of the roots of $63 \lambda^{3}+434=0$ and $\lambda=0$. The computations show that $m_{1}=6$ for $\lambda \neq 0$ and $m_{1}=0$ for $\lambda=0$. Thus, Method 6 outputs

$$
\operatorname{mult}((\lambda:-\lambda: 1))= \begin{cases}1 & \text { if } \lambda \neq 0 \\ 3 & \text { if } \lambda=0\end{cases}
$$

We observe that $\mathfrak{g}^{\mathcal{H}(0)} \neq 1$. So, $\mathcal{S}_{3}$ is a cone with vertex $\mathcal{H}(0)=(1,-45 /$ $124,0)$.

- Line $s+((1 / 2) \sqrt{-3}-1 / 2) t=0$. Then, $\mathcal{Q}(\lambda)=\left(-\left(\frac{1}{2} \sqrt{-3}-\frac{1}{2}\right) \lambda, \lambda\right)$ and $\mathcal{H}(\lambda)=\mathcal{P}_{3}(\mathcal{Q}(\lambda))$; we observe that $\mathcal{H}(\lambda)$ is not defined for the roots of $3 \lambda^{3}-124 \imath \sqrt{3}=0$. For $\mathcal{H}(\lambda)$ all steps specialize properly with the exception of $\lambda=0$, for the roots of $-126 \imath \sqrt{3} \lambda^{3}-588 \lambda^{3}+868 \imath \sqrt{3}=0$, and for the roots of $267 \lambda^{3}-2531 \imath \sqrt{3}=0$. The computations show that $m_{1}=0$ for $\lambda=0$ and $m_{1}=6$ for all $3 \lambda^{3}-124 \imath \sqrt{3} \neq 0$ with $\lambda \neq 0$. Thus, Method 6 outputs that

$$
\operatorname{mult}\left(\left(-\left(\frac{1}{2} \sqrt{-3}-\frac{1}{2}\right) \lambda: \lambda: 1\right)\right)= \begin{cases}1 & \text { if } \lambda \neq 0 \text { and } 3 \lambda^{3}-124 \imath \sqrt{3} \neq 0 \\ 3 & \text { if } \lambda=0\end{cases}
$$

Observe that, for $\lambda=0$, we get again the vertex of $\mathcal{S}_{3}$.

- Line $s-((1 / 2) \sqrt{-3}+1 / 2) t=0$. Reasoning as above we get

$$
\operatorname{mult}\left(\left(\left(\frac{1}{2} \sqrt{-3}+\frac{1}{2}\right) \lambda: \lambda: 1\right)\right)= \begin{cases}1 & \text { if } \lambda \neq 0 \text { and } 3 \lambda^{3}+124 \imath \sqrt{3} \neq 0 \\ 3 & \text { if } \lambda=0 .\end{cases}
$$

Observe that, for $\lambda=0$, we get again the vertex of $\mathcal{S}_{3}$.

So, we have computed the multiplicity for affine parameter values in $\Delta_{4} \backslash \Delta_{3}$. 


\section{[Execution of Method 8 ends, and hence Step 5 of the algorithm]}

In Step 6 , since $\left(\Delta_{4} \cap \Delta_{3}\right) \backslash \Delta_{2} \neq \emptyset$ we go to Step 6.2 where we compute the multiplicity, by using $\mathcal{P}_{2}(s, t)$, of the finitely many affine parameter values that we have not analyze yet. We get

$$
\begin{aligned}
& \operatorname{mult}\left(\left(-\left(\frac{1}{2} \sqrt{-3}-\frac{1}{2}\right) \lambda: \lambda: 1\right)\right)=1 \quad \text { if } 3 \lambda^{3}-124 \imath \sqrt{3}=0, \\
& \operatorname{mult}\left(\left(\left(\frac{1}{2} \sqrt{-3}+\frac{1}{2}\right) \lambda: \lambda: 1\right)\right)=1 \quad \text { if } 3 \lambda^{3}+124 \imath \sqrt{3}=0 .
\end{aligned}
$$

Now, since $\Delta_{4} \cap \Delta_{3} \cap \Delta_{2}=\emptyset$ go skip Step 7 and we go to Step 8. So, we have finished the analysis of the affine points, and we start with the study of the points at infinity. In Step 8 of the main algorithm, we observe that $\mathfrak{p}_{4}(0,1,0) \neq 0$. Thus, we compute $\operatorname{mult}\left(\mathcal{P}_{4}^{\mathrm{t}}(0,0), \mathcal{S}_{4}\right)$ by applying Method 6 . One gets that $m_{1}:=$ $\operatorname{deg}\left(\Phi_{2,3}\left(\mathcal{P}_{4}^{\mathrm{t}}(0,0)\right)\right)=6$. So,

$$
\operatorname{mult}((0: 1: 0))=\operatorname{mult}\left(\mathcal{P}_{4}^{\mathrm{t}}(0,0), \mathcal{S}_{4}\right)=1 \text {. }
$$

In Step 9, we observe that $\mathfrak{p}_{4}(1, \lambda, 0)=1+\lambda^{3}$. Moreover, we get that $\mathcal{E}^{*}=\emptyset$, and $\mathcal{E}^{* *}=\left\{(1, \lambda) \mid 1+\lambda^{3}=0\right\}$. Thus, in Step 10, we analyze $(1:-1: 0)$ and $(1: 1 / 2(1 \pm \imath \sqrt{3}): 0)$. Using $\mathcal{P}_{1}^{\mathfrak{s}}(s, t)$ we get that all are simple points. In Step 11, we apply Method 8 with input: $\mathcal{S}_{4}, \mathcal{P}_{4}^{\mathfrak{s}}(s, t), A_{0}=(1,1,1), n_{1}=9, n_{2}=3$, and $\Gamma=\left\{(1, \lambda) \mid 1+\lambda^{3} \neq 0\right\}$. We get

$$
\operatorname{mult}((1: \lambda: 0))=\operatorname{mult}\left(\mathcal{P}_{4}^{\mathfrak{s}}(\lambda, 0), \mathcal{S}_{4}\right)=1 .
$$

Note that all points at infinity are simple. Summarizing $\mathbb{P}^{2}(\mathbb{C})$ is decomposed as $\mathbb{P}^{2}(\mathbb{C})=\bigcup_{k=1}^{3} \mathfrak{S}_{k}$, where

(1) $\mathfrak{S}_{2}=\left\{\left(\frac{1}{2}(-1 \pm \sqrt{3} \imath) \mathfrak{t}: \mathfrak{t}: 1\right) \mid \mathfrak{t} \neq 0\right\}$,

(2) $\mathfrak{S}_{3}=\{(0: 0: 1)\}$,

(3) $\mathfrak{S}_{1}=\mathbb{P}^{2}(\mathbb{C}) \backslash\left(\mathfrak{S}_{2} \cup \mathfrak{S}_{3}\right)$.

\section{ACKNOWLEDGEMENT}

We thank the referee for his/her comments and suggestions, since they have helped to improve this paper.

\section{REFERENCES}

[1] Falai Chen, Wenping Wang, and Yang Liu, Computing singular points of plane rational curves, J. Symbolic Comput. 43 (2008), no. 2, 92-117, DOI 10.1016/j.jsc.2007.10.003. MR2357078 (2009a:14035)

[2] Joe Harris, Algebraic Geometry: A First Course, Graduate Texts in Mathematics, vol. 133, Springer-Verlag, New York, 1995. Corrected reprint of the 1992 original. MR 1416564 (97e:14001)

[3] Xiaohong Jia, Falai Chen, and Jiansong Deng, Computing self-intersection curves of rational ruled surfaces, Comput. Aided Geom. Design 26 (2009), no. 3, 287-299, DOI 10.1016/j.cagd.2008.09.005. MR2493078(2010a:65032)

[4] Hyungju Park, Effective computation of singularities of parametric affine curves, J. Pure Appl. Algebra 173 (2002), no. 1, 49-58, DOI 10.1016/S0022-4049(02)00017-8. MR1912959 (2003f:14068)

[5] Sonia Pérez-Díaz, Computation of the singularities of parametric plane curves, J. Symbolic Comput. 42 (2007), no. 8, 835-857, DOI 10.1016/j.jsc.2007.06.001. MR2345839 (2008g:14116)

[6] Sonia Pérez-Díaz and J. Rafael Sendra, Computation of the degree of rational surface parametrizations, J. Pure Appl. Algebra 193 (2004), no. 1-3, 99-121, DOI 10.1016/j.jpaa.2004.02.011. MR2076380 (2005d:14090) 
[7] Sonia Pérez-Díaz and J. Rafael Sendra, Partial degree formulae for rational algebraic surfaces, ISSAC'05, ACM, New York, 2005, pp. 301-308, DOI 10.1145/1073884.1073926. MR2280561

[8] Sonia Pérez-Díaz and J. Rafael Sendra, A univariate resultant-based implicitization algorithm for surfaces, J. Symbolic Comput. 43 (2008), no. 2, 118-139, DOI 10.1016/j.jsc.2007.10.001. MR2357079(2009b:14112)

[9] S. Pérez-Díaz, J. R. Sendra, and C. Villarino, A first approach towards normal parametrizations of algebraic surfaces, Internat. J. Algebra Comput. 20 (2010), no. 8, 977-990, DOI 10.1142/S0218196710005972. MR2747411 (2011m:68303)

[10] R. Rubio, J. M Serradilla, and M. P. Vélez, Detecting real singularities of a space curve from a real rational parametrization, J. Symbolic Comput. 44 (2009), no. 5, 490-498, DOI 10.1016/j.jsc.2007.09.002. MR2499924 (2010h:14095)

[11] J. Rafael Sendra and Franz Winkler, Tracing index of rational curve parametrizations, Comput. Aided Geom. Design 18 (2001), no. 8, 771-795, DOI 10.1016/S0167-8396(01)00069-3. MR:1857997(2002h:65022)

[12] J. Rafael Sendra, Franz Winkler, and Sonia Pérez-Díaz, Rational Algebraic Curves, Algorithms and Computation in Mathematics, vol. 22, Springer, Berlin, 2008. A computer algebra approach. MR2361646 (2009a:14073)

[13] Igor R. Shafarevich, Basic Algebraic Geometry. 1. Varieties in Projective Space, 2nd ed., Springer-Verlag, Berlin, 1994. Translated from the 1988 Russian edition and with notes by Miles Reid. MR1328833(95m:14001)

[14] F. Winkler, Polynomial Algorithms in Computer Algebra, Texts and Monographs in Symbolic Computation, Springer-Verlag, Vienna, 1996. MR1408683 (97j:68063)

[15] O. Zariski, P. Samuel, Commutative Algebra, Volume I. Graduate Texts in Mathematics, vol. 28, Springer-Verlag, 1975.

Dpto. de Física y Matemáticas, Universidad de Alcalá, E-28871 Madrid, Spain

E-mail address: sonia.perez@uah.es

Dpto. de Física y Matemáticas, Universidad de Alcalá, E-28871 Madrid, Spain

E-mail address: rafael.sendra@uah.es

Dpto. de Física y Matemáticas, Universidad de Alcalá, E-28871 Madrid, Spain

E-mail address: carlos.villarino@uah.es 